\title{
Bounds on Herglotz functions and fundamental limits of broadband passive quasi-static cloaking
}

\author{
Maxence Cassier \\ Department of Mathematics, University of Utah, Salt Lake City UT 84112, USA \\ (cassier@math.utah.edu) \\ Graeme W. Milton \\ Department of Mathematics, University of Utah, Salt Lake City UT 84112, USA \\ (milton@math.utah.edu)
}

\begin{abstract}
Using a sum rule, we derive new bounds on Herglotz functions that generalize those given in [31, 12. These bounds apply to a wide class of linear passive systems such as electromagnetic passive materials. Among these bounds, we describe the optimal ones and also discuss their meaning in various physical situations like in the case of a transparency window, where we exhibit sharp bounds. Then, we apply these bounds in the context of broadband passive cloaking in the quasi-static regime to negatively answer the following challenging question: is it possible to construct a passive cloaking device that cloaks an object over a whole frequency band? Our rigorous approach, although limited to quasistatics, gives quantitative limitations on the cloaking effect over a finite frequency range by providing inequalities on the polarizabilty tensor associated with the cloaking device. We emphasize that our results hold for a cloak or object of any geometrical shape.
\end{abstract}

Keywords: Invisibility, passive cloaking, Herglotz and Stieltjes functions, dispersive Maxwell's equations, quasi-statics.

\section{Introduction}

For many years it has been know that there exist inclusions that can be invisible to certain applied fields. These are generally known as neutral inclusions (see, for example, the references in Section 7.11 in [55], and see also the more recent citations of these papers) and references therein) and a specific example are the invisible bodies of Kerker [38], that are in fact coated confocal ellipsoids, which are invisible to long wavelength fields. More intriguing are the cylindrical shells of NIcorovici, McPhedran, and Milton [69] having (at a fixed frequency) a relative permittivity of -1 , surrounded by material having a reative permittivity of 1 , that are invisible to any polynomial quastistatic applied field, and the coated spheres of Alú and Engheta [1, that are invisible at a specific frequency. For conductivity and fixed frequency electromagnetism Tartar (in a private communication to Kohn and Vogelius [41]) and Dolin [21] recognised that one could create a wide class of invisible inclusions with anisotropic moduli by transformation conductivity and transformation optics. A subsequent key idea of Greenleaf, Lassas, and 
Uhlmann [26, 27] was that one could create a cloak for conductivity (and hence single frequency quasi-statics) by using singular transformations that created a "quiet zone" where no field penetrated, and hence where one could place an object without disturbing the surrounding current field. The next development was the recognition by Milton and Nicorovici [57] that cloaking due to anomalous resonance could cloak (at least in two-dimensional quasi-statics, though some results were also obtained in three-dimensions and at finite frequency) an arbitary finite number of polarizable dipoles: this had the fascinating feature that the cloaking region lay outside the cloaking device. It was perhaps the first paper where the word cloaking appeared in the scientific literature, outside computer science. Shortly afterwards, papers appeared by Leonhardt [44] and Pendry, Schurig, and Smith [83] using transformation ideas to obtain cloaking for geometric optics and Maxwell's equations at fixed frequencies. These three papers, of Milton and Nicorovici, Leonhardt, and Pendry, Schurig and Smith generated considerable media attention, and also stimulated a lot of subsequent scientific development, both on cloaking due to anomalous resonance [72, 16, 58, 71, 49, 15, 770, 40, 3, 4, 15, 39, 7, 63, 50, 46, 65, 80, 64] and on transformation based cloaking (see, for example, the reviews [2] and [25]). Other sorts of cloaking were developed too, including cloaking due to complementary media [42], that has anomalous resonance as its mechanism [68, 67], and active cloaks [51, 28, 29, 30, 79, 74, 85, 75] where sources tailored to the incoming signal, and sometimes also tailored to the body to be cloaked [77, 78], create a cloak, yet do not significantly radiate. There is no theoretical difficulty in creating broadband active cloaks: each frequency can be cloaked separately and sources can then be designed that superimpose the contributions from the different frequencies. A good example is the cloaking of an object from an incoming pulse in the animation movies in [29].

Here our focus is on finding limitations to broadband cloaking for passive quasi-static cloaks. Unlike active cloaks which require energy sources to activate them, passive cloaks perform cloaking only by the physical properties of the passive material which constitutes the cloak. In the context of transformation based cloaking it has long been recognized that a cloak that guides waves around an object has the inherent limitation that a pulse signal (hence containing many frequencies) travelling on a ray cannot travel faster than the speed of light and therefore, if the ray goes around the body, the pulse will arrive later compared to a pulse that travels in a straight line at the speed of light. However we would like some more explicit quantitative bounds that limit cloaking, in particular over a specific frequency interval. Anomalous resonance uses materials with a negative dielectric constant and transformation based cloaks use materials with relative electrical permittivities (relative compared to the surrounding medium) less than one. Thus if the surrounding medium has the electrical permittivity of free space there should necessarily be some variation of the moduli with frequency, i.e., dispersion. While some experiments report broadband cloaking it is to emphasized that the surrounding medium is silicon, and this makes it possible to achieve a relative electrical permittivity that is less than 1 that is almost frequency independent. It seems that a clue to establishing broadband limitations to cloaking is to use bounds limiting the minimal dispersion in the component materials. For geometric optics Leonhardt and Tyc [45] show one can get broadband cloaking by ingeneous transformations from non-Euclidean geometries to Euclidean ones. (Such transformations are okay for geometric optics, but generally do not preserve the form of the time-harmonic Maxwell's equations.)

The main tool used to derive our bounds is to follow the idea developed in the analytic method introduced in [10, 53], justified in [54] and proved in [24]. In other words, to use the an- 
alytic properties of physical quantities (like the dielectric permittivity and the magnetic permeability in electromagnetism) which define the constitutive laws of the medium in the frequency domain. These properties are the counterpart of causality and passivity of time-dependent passive linear materials. Mathematically speaking, it is directly linked to the existence of a Herglotz and/or a Stieltjes function which characterizes the behavior of the system in the frequency domain [76, 18, 55, 92, 12. This analytic method under various forms has been widely applied to study physical properties of passive electromagnetic media in different contexts: to bound the dielectric permittivity with respect to the frequency [12, 31, to evaluate the resolution of a perfect lens on a finite bandwidth [47, to derive scattering limits as for instance upper bounds on the total extinction cross-section [84, 32, 52] or to provide quantitative limits to speed light propagation in dispersive media 90. In this paper, one wants to use such a method to derive bounds on the polarizability tensor associated with a cloaking device. This tensor is defined as a $3 \times 3$ complex-valued matrix function of the frequency [35, 55] which characterizes the main contribution of the far field of the scattered wave due to a cloaking device in the quasi-static regime of Maxwell's equations. Therefore if it vanishes at a frequency $\omega$, one says that the obstacle is cloaked at $\omega$ for a far observer. We prove in this paper that is not possible on a whole frequency band and derive inequalities to quantify this phenomenon.

Related to the question of broadband passive cloaking, we mention that Monticone and Alú [59] show that one cannot perform passive cloaking on the whole frequency spectrum by deriving a global bound on the scattering cross-section. More recently [60] they use electrical circuit analogies, to bound the scattering cross section over a finite frequency range for planar objects. Another interesting point was developed by Hashemi, Qiu, McCauley, Joannopoulos and Johnson [33] who demonstrate for the particular case of Lorentz dispersion models that broadband passive cloaking is limited by the obstacle characteristic size. Here, the bounds that we derive have the great advantage of neither assuming the geometrical shape of the object, or cloak, nor the dispersion models of the cloak. In fact the object could even lie outside the cloak. Moreover, they involve the size of the frequency bandwidth. While they are limited to quasistatics, they apply to cloaking due to anomalous resonance, transformation based cloaking, and cloaking due to complementary media and in fact to any quasi-static passive cloaking device.

The paper is organized as follows. In section 2, we first derive, using complex analysis, general bounds that are applicable to a broadband class of passive linear systems including electromagnetic passive media. More precisely, for an electromagnetic passive material, the standard notions of passivity and causality are introduced and this leads to four constraints on the dielectric permittivity and magnetic permeability behaviors seen as complex valued functions of the frequency. To develop our bounds in the general framework of linear passive system, we reformulate these four constraints as assumptions on a abstract complex-valued function $f$. Then, we briefly recall some basic notions on Stieltjes and Herglotz functions which are used throughout the paper. Our next step is to construct a Stieltjes and a Herglotz function associated with $f$. Afterwards, using the sum rules derived in [12] for Herglotz functions, we derive bounds parametrized by a set a of probability measures that generalized the bounds of [31, 12]. Then, we prove that among these bounds, the ones that are optimal are obtained using Dirac measures (see Theorem 14). Using such measures in the case of a transparency window (which physically means that the material is lossless on the considered frequency range), we recover a bound similar to the ones derived in [56, 91] which is sharp for Drude type models. We show that this last bound can be also easily established by another approach based on 
Kramers-Kronig relations. We finally explore the case of lossy material and recovers by our approach a bound similar to the ones derived in [31, 12. The section 3 of the paper is devoted to the applications of the previous bounds to the broadband passive cloaking question for the quasi-static approximation of Maxwell's equations. We first mathematically reformulate our cloaking problem in a rigorous functional framework and shows that the bounds derived in section 2 apply to the polarizability tensor associated with a passive cloaking device. Finally, we show that it is not possible to construct a passive cloak that achieves broadband cloaking on a finite range of frequencies and discuss the meaning of our bounds as fundamental limits of the cloaking effect in various situations like a transparency window or the general case of a lossy material.

\section{Bounds on Herglotz functions}

\subsection{Characterization of passive electromagnetic media}

In this subsection, one introduces the standard notions of causality and passivity for linear timedependent Maxwell's equations and their counterparts in the frequency domain. For simplicity, we are dealing here with an isotropic homogeneous material which fills a bounded domain $\Omega \subset$ $\mathbb{R}^{3}$, but one can derive such properties in the general setting of anisotropic and inhomogeneous materials. For more details, we refer to [76, 43, 18, 55, 92, 90].

We denote respectively by $\mathbf{D}$ and $\mathbf{B}$ the electric and magnetic inductions, by $\mathbf{E}$ and $\mathbf{H}$ the electric and magnetic fields, the evolution of $(\mathbf{E}, \mathbf{D}, \mathbf{H}, \mathbf{B})$ in $\Omega$ is governed (on the absence of a current density source) by the macroscopic Maxwell's equations:

$$
\partial_{t} \mathbf{D}-\nabla \times \mathbf{H}=0 \quad \text { and } \quad \partial_{t} \mathbf{B}+\nabla \times \mathbf{E}=0,
$$

which must be supplemented by the constitutive laws of the material involving two additional unknowns the electric and magnetic polarizations $\mathbf{P}$ and $\mathbf{M}$ :

$$
\mathbf{D}=\varepsilon_{0} \mathbf{E}+\mathbf{P} \text { with } \mathbf{P}=\varepsilon_{0} \chi_{E} \star_{t} \mathbf{E} \text { and } \mathbf{B}=\mu_{0} \mathbf{H}+\mathbf{M} \text { with } \mathbf{M}=\mu_{0} \chi_{M} \star_{t} \mathbf{H} .
$$

The constants $\varepsilon_{0}$ and $\mu_{0}$ stand here for the permittivity and permeability of the vacuum. The constitutive laws express the relations between $(\mathbf{D}, \mathbf{E})$ and $(\mathbf{B}, \mathbf{H})$ via a convolution in time with the electrical and magnetic susceptibility $\chi_{E}$ and $\chi_{M}$, defined here as scalar time-dependent functions which characterize the electromagnetic behavior of the material.

We assume here for simplicity that $\chi_{E}$ and $\chi_{M} \in L^{1}\left(\mathbb{R}_{t}\right)$, the space of integrable functions with respect to the time variable. In a more general setting, one can consider them as tempered distributions (see [18, 92]). We suppose also that $\mathbf{E}, \mathbf{H}, \partial_{t} \mathbf{E}$ and $\partial_{t} \mathbf{H}$ are in $\mathbf{L}^{2}\left(\mathbb{R}_{t}, \mathbf{L}^{2}(\Omega)\right)$. Hence, as $(\mathbf{E}, \mathbf{D}, \mathbf{H}, \mathbf{B})$ satisfy $(2.1)$ and $(2.2)$, one deduces with such hypothesis that $\mathbf{D}, \mathbf{B}, \partial_{t} \mathbf{D}, \partial_{t} \mathbf{B}, \nabla \times \mathbf{E}$ and $\nabla \times \mathbf{H}$ are also in $\mathbf{L}^{2}\left(\mathbb{R}_{t}, \mathbf{L}^{2}(\Omega)\right)$. In this functional framework, one introduces four standard properties which model the constitutive laws of electromagnetic passive linear systems in the frequency domain.

- A material is said to be causal if the fields $\mathbf{E}(\cdot, t)$ and $\mathbf{H}(\cdot, t)$ cannot influence the inductions $\mathbf{D}\left(\cdot, t^{\prime}\right)$ and $\mathbf{B}\left(\cdot, t^{\prime}\right)$ for $t^{\prime}<t$. This condition implies that the functions $\chi_{E}$ and $\chi_{M}$ are supported in $\mathbb{R}^{+}$. To see the counterpart of the causality in the frequency domain, one defines the LaplaceFourier transform of a function of $f \in L^{1}\left(\mathbb{R}_{t}\right)$ supported in $\mathbb{R}^{+}$by

$$
\hat{f}(\omega)=\int_{\mathbb{R}^{+}} f(t) \mathrm{e}^{\mathrm{i} \omega t} \mathrm{~d} t, \forall \omega \in \operatorname{cl} \mathbb{C}^{+},
$$


where $\operatorname{cl} \mathbb{C}^{+}:=\{\omega \in \mathbb{C} \mid \operatorname{Im}(\omega) \geq 0\}$ stands for topological closure of the complex upper halfplane $\mathbb{C}^{+}:=\{\omega \in \mathbb{C} \mid \operatorname{Im}(\omega)>0\}$. We point out that the Laplace-Fourier transform coincides with the Fourier transform for real frequency $\omega$, that is why we use in the following the same notation for both transforms. Classically, applying the Fourier transform to 2.2 for real $\omega$ leads to the well-known expression for the constitutive laws 2.2 in the frequency domain:

$\hat{\mathbf{D}}(\omega)=\varepsilon(\omega) \hat{\mathbf{E}}(\omega)$ with $\varepsilon(\omega)=\varepsilon_{0}\left(1+\hat{\chi}_{E}(\omega)\right)$ and $\hat{\mathbf{B}}(\omega)=\mu(\omega) \hat{\mathbf{H}}(\omega)$ with $\mu(\omega)=\mu_{0}\left(1+\hat{\chi}_{M}(\omega)\right)$,

where $\varepsilon(\omega)$ and $\mu(\omega)$ stand for the dielectric permittivity and the magnetic permeability of the material. Now, as $\chi_{E}$ and $\chi_{M} \in L^{1}\left(\mathbb{R}_{t}\right)$ are compactly supported in $\mathbb{R}^{+}$, one deduces easily that their Laplace Fourier transforms $\hat{\chi}_{E}$ and $\hat{\chi}_{M}$ are analytic in the upper half-plane $\mathbb{C}^{+}$and continuous on $\mathrm{cl} \mathbb{C}^{+}$. Thus, $\varepsilon=\varepsilon_{0}\left(1+\hat{\chi}_{E}\right)$ and $\mu=\mu_{0}\left(1+\hat{\chi}_{M}\right)$ share the same regularity.

- Furthermore, by applying the Riemann-Lebesgue theorem (since $\chi_{E}$ and $\chi_{M} \in L^{1}\left(\mathbb{R}_{t}\right)$ ), one has that $\hat{\chi}_{E}$ and $\hat{\chi}_{M}$ tend to 0 , as $|\omega| \rightarrow \infty$ in $\operatorname{cl} \mathbb{C}^{+}$. Hence, we have

$$
\varepsilon(\omega) \rightarrow \varepsilon_{0} \text { and } \mu(\omega) \rightarrow \mu_{0}, \text { as }|\omega| \rightarrow \infty \text { in } \mathrm{cl} \mathbb{C}^{+} .
$$

In other words, the material behaves as the vacuum for high frequencies.

- As $\chi_{E}$ and $\chi_{M}$ are real functions, it implies that their Laplace-Fourier transforms defined by (2.3) satisfisfy the following "symmetry" relations:

$$
\hat{\chi}_{E}(-\bar{\omega})=\overline{\hat{\chi}_{E}(\omega)} \text { and } \hat{\chi}_{M}(-\bar{\omega})=\overline{\hat{\chi}_{M}(\omega)}, \quad \forall \omega \in \operatorname{cl} \mathbb{C}^{+}
$$

and thus the same relation holds for the functions $\varepsilon$ and $\mu$.

- The passivity assumption is expressed as the following (see [12, 18, 43, 55, 90]):

$$
\mathcal{E}_{a}(t)=\int_{-\infty}^{t} \int_{\Omega} \partial_{t} \mathbf{D}(\mathbf{x}, s) \cdot \mathbf{E}(\mathbf{x}, s)+\partial_{t} \mathbf{B}(\mathbf{x}, s) \cdot \mathbf{H}(\mathbf{x}, s) \mathrm{d} \mathbf{x} \mathrm{d} s \geq 0, \forall t \in \mathbb{R}
$$

and holds for any fields $(\mathbf{E}, \mathbf{H})$ such that

$$
\mathbf{E}, \mathbf{H} \in \mathbf{L}^{2}\left(\mathbb{R}_{t}, \mathbf{L}^{2}(\Omega)\right) \text { and } \partial_{t} \mathbf{E}, \partial_{t} \mathbf{H} \in \mathbf{L}^{2}\left(\mathbb{R}_{t}, \mathbf{L}^{2}(\Omega)\right) .
$$

This assumption imposes physically that at each time, the amount of electromagnetic energy $\mathcal{E}_{a}(t)$ transferred to the material by Joule effect or absorption, that is by electric and/or magnetic loss is positive. By virtue of the Plancherel theorem and the constitutive laws $(2.2)$, the passivity assumption (2.5) applied to $t=\infty$ yields the following inequality in the frequency domain:

$\mathcal{E}_{a}(\infty)=\frac{1}{2 \pi} \operatorname{Re}\left(\int_{\mathbb{R}} \int_{\Omega}-\mathrm{i} \omega\left(\varepsilon_{0}\left(1+\hat{\chi}_{E}(\omega)\right)|\hat{\mathbf{E}}(\mathbf{x}, \omega)|^{2}+\mu_{0}\left(1+\hat{\chi}_{M}(\omega)\right)|\hat{\mathbf{H}}(\mathbf{x}, \omega)|^{2}\right) \mathrm{d} \mathbf{x} \mathrm{d} \omega\right) \geq 0$

which can be rewritten as

$$
\mathcal{E}_{a}(\infty)=\frac{1}{2 \pi} \int_{\mathbb{R}} \int_{\Omega} \omega \operatorname{Im} \varepsilon(\omega)|\hat{\mathbf{E}}(\mathbf{x}, \omega)|^{2}+\omega \operatorname{Im} \mu(\omega)|\hat{\mathbf{H}}(\mathbf{x}, \omega)|^{2} \mathrm{~d} \mathbf{x} \mathrm{d} \omega \geq 0 .
$$

Hence, as the last inequality holds for any fields $\mathbf{E}$ and $\mathbf{H}$ which satisfy the conditions $(2.6)$ in the time-domain, it is straightforward (using a proof by contradiction) to show that it implies 
that $\omega \operatorname{Im} \varepsilon(\omega) \geq 0$ and $\omega \operatorname{Im} \mu(\omega) \geq 0$, for all real frequency $\omega$. These latter conditions turn out to be equivalent, by (2.4), to

$$
\operatorname{Im} \varepsilon(\omega) \geq 0 \text { and } \operatorname{Im} \mu(\omega) \geq 0, \forall \omega \in \mathbb{R}^{+},
$$

that is referred to the characterization of passivity in the frequency domain [43, 18, 55,

Reciprocally, the condition (2.8) and the fact that $\varepsilon$ and $\mu$ are bounded, continuous func-

tions (since $\chi_{E}$ and $\chi_{M} \in L^{1}\left(\mathbb{R}_{t}\right)$ ) satisfying 2.4 on $\mathbb{R}_{\omega}$ imply, in particular, that inequality 2.7 holds for any $\hat{\mathbf{E}}, \hat{\mathbf{H}} \in \mathbf{L}^{2}\left(\mathbb{R}_{\omega}, \mathbf{L}^{2}(\Omega)\right)$ such that $\mathbf{E}$ and $\mathbf{H} \in \mathcal{D}\left((-\infty, t), \mathbf{L}^{2}(\Omega)\right.$ ) (where $\mathcal{D}\left((-\infty, t), \mathbf{L}^{2}(\Omega)\right)$ refers to the space of bump functions of $(-\infty, t)$ valued in $\left.\mathbf{L}^{2}(\Omega)\right)$. Hence by Plancherel's theorem, one obtains that passivity assumption (2.5) holds at any fixed time $t \in \mathbb{R}$ and for any $\mathbf{E}$ and $\mathbf{H} \in \mathcal{D}\left((-\infty, t), \mathbf{L}^{2}(\Omega)\right)$. Finally, one extends by a density argument this relation to any fields $\mathbf{E}$ and $\mathbf{H}$ satisfying (2.6). Thus, (2.8) is equivalent to (2.5).

The aim of this section is to derive in a general framework a bound for a function $f: \mathrm{cl} \mathbb{C}^{+} \mapsto$ $\mathbb{C}$ which satisfies the following hypotheses:

- H1: $f$ is analytic on the upper half plane $\mathbb{C}^{+}$and continuous on $\mathrm{cl} \mathbb{C}^{+}$,

- $\mathrm{H} 2: f(z) \rightarrow f_{\infty}>0$, when $|z| \rightarrow \infty$ in $\mathrm{cl}^{+}$,

- H3: $f$ satisfies $f(-\bar{z})=\overline{f(z)}, \quad \forall z \in \mathrm{cl} \mathbb{C}^{+}$,

- H4: $\operatorname{Im} f(z) \geq 0$ for all $z \in \mathbb{R}^{+}$(passivity).

described above for $f=\varepsilon$ or $f=\mu$ as function of the frequency $\omega$. More generally, these hypotheses characterize the frequency behavior of passive linear systems [92, 12]. They are satisfied by the permittivity and the permeability but also by other physical quantities such as the polarizability tensor in the quasi-static regime (as it will be proved in subsection 3.2), the acoustic [73] and electromagnetic [76, 32] forward scattering amplitudes and the shear and bulk modulus in elasticity [13. Thus, the bounds we develop in this first part, in this general setting, apply to all these physical parameters and constrain their behavior in the frequency domain.

\subsection{Review of some Herglotz and Stieltjes functions properties}

Mathematically, the hypotheses H1-4 on the function $f$ are linked to the existence of a Stieltjes and a Herglotz function associated with $f$. Stieltjes and Herglotz functions have been extensively used in the study of electromagnetic materials' behavior (see for instance [56, 55, 31, 12, 90]). The aim of this subsection is to recall briefly some properties about these functions that we use in the following to derive our bounds. For more details, we refer to [62, $8,123,9$, 12,

Definition 1. An analytic function $h: \mathbb{C}^{+} \rightarrow \mathbb{C}$ is a Herglotz function (also called Pick or Nevanlinna function) if

$$
\operatorname{Im} h(z) \geq 0, \forall z \in \mathbb{C}^{+} .
$$

A particular and useful property of Herglotz functions is the following representation theorem due to Nevanlinna [62]. 
Theorem 2. A necessary and sufficient condition for $h$ to be a Herglotz function is given by the following representation:

$$
h(z)=\alpha z+\beta+\int_{\mathbb{R}}\left(\frac{1}{\xi-z}-\frac{\xi}{1+\xi^{2}}\right) \operatorname{dm}(\xi), \text { for } \operatorname{Im}(z)>0,
$$

where $\alpha \in \mathbb{R}^{+}, \beta \in \mathbb{R}$ and $\mathrm{m}$ is a positive regular Borel measure for which $\int_{\mathbb{R}} \operatorname{dm}(\xi) /\left(1+\xi^{2}\right)$ is finite. In particular if the integral $\int_{\mathbb{R}} \xi \operatorname{dm}(\xi) /\left(1+\xi^{2}\right)$ is also finite, then we can rewrite the relation (2.9) as:

$$
h(z)=\alpha z+\gamma+\int_{\mathbb{R}} \frac{\operatorname{dm}(\xi)}{\xi-z} \quad \text { with } \gamma=\beta-\int_{\mathbb{R}} \frac{\xi \operatorname{dm}(\xi)}{1+\xi^{2}} \in \mathbb{R} .
$$

Moreover, for a given Herglotz function $h$, the triple $(\alpha, \beta, \mathrm{m})$ is uniquely defined by the following corollary.

Corollary 3. Let $h$ be a Herglotz function defined by its representation (2.9), then we have:

$$
\begin{gathered}
\alpha=\lim _{y \rightarrow+\infty} \frac{h(\mathrm{i} y)}{\mathrm{i} y}, \beta=\operatorname{Re} h(\mathrm{i}), \\
\text { and } \forall[a, b] \subset \mathbb{R}, \frac{\mathrm{m}([a, b])+\mathrm{m}((a, b))}{2}=\lim _{y \rightarrow 0^{+}} \frac{1}{\pi} \int_{a}^{b} \operatorname{Im} h(x+\mathrm{i} y) \mathrm{d} x .
\end{gathered}
$$

We now introduce for any $\theta \in(0, \pi / 2)$ the Stolz domain $D_{\theta}$ defined by:

$$
D_{\theta}=\{z \in \mathbb{C} \mid \theta \leq \arg (z) \leq \pi-\theta\} .
$$

The representation theorem 2.9 implies (see [12]) that a Herglotz function satisfies the following asymptotics in $D_{\theta}$ for all $\theta \in(0, \pi / 2)$ :

$$
h(z)=-\mathrm{m}(\{0\}) z^{-1}+o\left(z^{-1}\right) \text { as }|z| \rightarrow 0 \text { and } h(z)=\alpha z+o(z) \text { as }|z| \rightarrow+\infty .
$$

In other words, an Herglotz function grows at most as rapidly $z$ when $|z|$ tends to $+\infty$ and cannot be more singular than $z^{-1}$ when $|z|$ tends to 0 .

We will conclude this review of Herglotz functions by a last identity: the so-called sum rule (see [12]) which is a fundamental tool to derive quantitative bounds on passive systems.

Proposition 4. Let $h$ be a Herglotz function which admits the following asymptotic expansions in $D_{\theta}$ for all $\theta \in(0, \pi / 2)$ :

$$
\begin{gathered}
h(z)=a_{-1} z^{-1}+o\left(z^{-1}\right) \quad \text { as }|z| \rightarrow 0, \\
\text { and } \quad h(z)=b_{-1} z^{-1}+o\left(z^{-1}\right) \quad \text { as }|z| \rightarrow+\infty .
\end{gathered}
$$

with $a_{-1}$ and $b_{-1} \in \mathbb{R}$. Then the following identity holds

$$
\lim _{\eta \rightarrow 0^{+}} \lim _{y \rightarrow 0^{+}} \frac{1}{\pi} \int_{\eta<|x|<\eta^{-1}} \operatorname{Im} h(x+\mathrm{i} y) \mathrm{d} x=a_{-1}-b_{-1} .
$$


We now introduce Stieltjes functions: another famous class of analytic functions, closely related to Herglotz functions.

Definition 5. A Stieltjes function is an analytic function $g: \mathbb{C} \backslash \mathbb{R}^{-} \rightarrow \mathbb{C}$ which satisfies:

$$
\operatorname{Im} g(z) \leq 0 \quad \forall z \in \mathbb{C}^{+} \text {and } g(x) \geq 0 \text { for } x>0 .
$$

Like Herglotz functions, Stieltjes functions are characterized by a representation theorem.

Theorem 6. A necessary and sufficient condition for $g$ to be a Stieltjes function is given by the following representation:

$$
g(z)=\alpha+\int_{\mathbb{R}^{+}} \frac{\mathrm{dm}(\xi)}{\xi+z} \quad \forall z \in \mathbb{C} \backslash \mathbb{R}^{-},
$$

where $\alpha=\lim _{|z| \rightarrow+\infty} g(z) \in \mathbb{R}^{+}$and $\mathrm{m}$ is a positive regular Borel measure, uniquely defined, for which $\int_{\mathbb{R}^{+}} \operatorname{dm}(\xi) /(1+\xi)$ is finite.

Remark 7. An easy connection can be made between Herglotz and Stieljes function. Thanks to the representation Theorems 2 and 6, we note that if $g$ is a Stieltjes function, the function $h$ defined by $h(z)=g(-z)$ is an Herglotz function whose measure $\mathrm{m}$ has a support included in $\mathbb{R}^{+}$in the relation (2.9). Another connection between Herglotz and Stieltjes functions is given in the next subsection by Corollary 10.

\subsection{Construction of a Stieltjes function associated with $f$}

In this paragraph, we construct a Stieltjes function associated with the function $f$. For that, we first establish with the following lemma some information about the sign of the imaginary part of the function $f$.

Lemma 8. If a function $f$ satisfies the hypotheses H1-4, then

$$
\pm \operatorname{Im} f(z) \geq 0, \forall z \in \mathbb{C}^{+} \text {such that } \pm \operatorname{Re} z \geq 0 .
$$

Moreover, if $f$ is not a constant function, the inequalities 2.13) are strict as soon as $\operatorname{Re} z \neq 0$.

Proof. Let $\mathcal{O}$ denote the open set $\mathcal{O}=\left\{z \in \mathbb{C}^{+} \mid \operatorname{Re}(z)>0\right\}$. By virtue of H1, $\operatorname{Im} f$ is an harmonic function on $\mathcal{O}$ that is continuous on $\mathrm{cl} \mathcal{O}$. $\mathrm{H} 3$ and $\mathrm{H} 4$ imply respectively that $f$ is real on the imaginary axis and that $\operatorname{Im} f(z) \geq 0$ on the positive real axis, thus we get that $\operatorname{Im} f(z) \geq 0$ on the boundary $\partial \mathcal{O}$ of $\mathcal{O}$. Moreover, from $\mathrm{H} 2$ it follows that $\operatorname{Im} f(z) \rightarrow 0$ as $|z| \rightarrow \infty$ in $\mathrm{cl} \mathcal{O}$. All these conditions allow us to apply the maximum principle on the function $\operatorname{Im} f$ in the unbounded domain $\mathcal{O}$ (see Corollary 4 p 246 of [20]) which yields the inequality 2.13 for $\operatorname{Re}(z) \geq 0$. The inequality 2.13 for $\operatorname{Re}(z) \leq 0$ is then deduced by using H3.

In the case where $f$ is not a constant function, by contradiction, if there exists a $z_{0} \in \mathbb{C}^{+}$ with a positive real part such that $\operatorname{Im} f\left(z_{0}\right)=0$, then by the open mapping theorem the image by $f$ of an open ball $B\left(z_{0}, \delta\right) \subset \mathcal{O}$ is an open set of $\mathbb{C}$ which contains a real number $f\left(z_{0}\right)$ and therefore some points with a negative imaginary part. This contradicts (2.13). Finally, by using H3, one obtains also that $\operatorname{Im} f(z)<0$ for $\operatorname{Re}(z)<0$. 

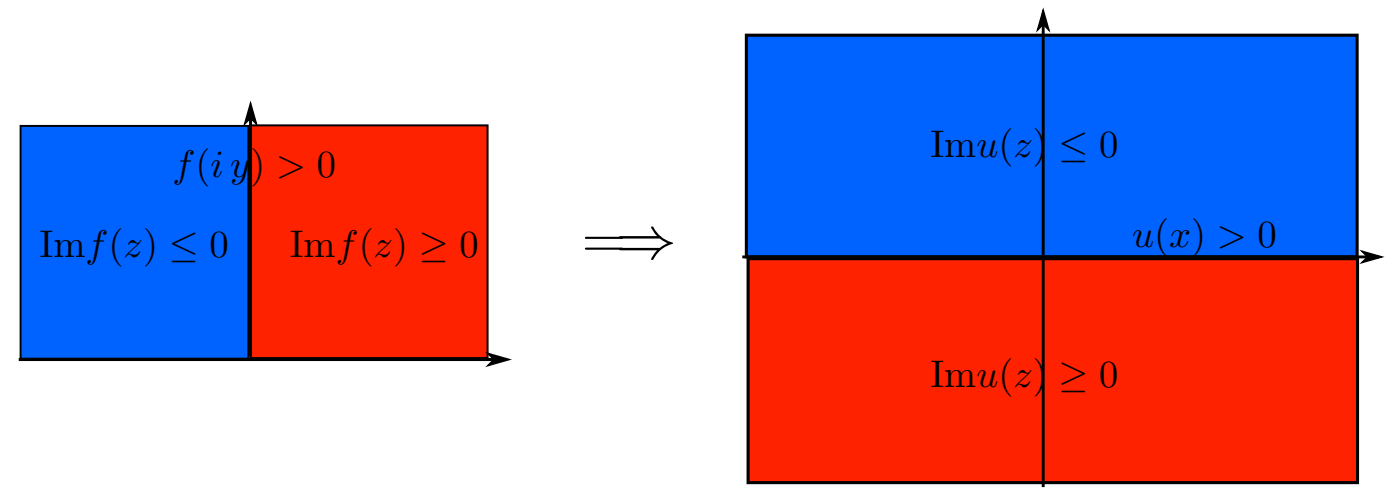

Figure 1: Sign of the imaginary part of the functions $f$ (left) and $u$ (right).

To construct a Stieltjes function associated with $f$, we will follow the idea proposed by the authors of [56]. For that purpose, we define the complex root by

$$
\sqrt{z}=|z|^{\frac{1}{2}} e^{\mathrm{i} \arg z / 2} \quad \text { if } \arg z \in(0,2 \pi)
$$

and extend it on the branch cut $\mathbb{R}^{+}$by its limit from the upper-half plane, in other words the square root of positive real number $x$ is given by $\sqrt{x}=|x|^{\frac{1}{2}}$.

Theorem 9. If $f$ satisfies the hypotheses H1-4, then the function $u$ defined by

$$
u(z):=f(\sqrt{-z}), \forall z \in \mathbb{C}
$$

is a Stieltjes function which is positive on $\mathbb{R}^{+*}$.

Proof. The definition of the complex square root and the hypothesis H1 directly imply that $u$ is analytic on $\mathbb{C} \backslash \mathbb{R}^{-}$. Moreover, using the property H3 and the Lemma 8 , we get that

$$
u\left(\mathbb{C}^{+}\right)=f\left(\left\{z \in \mathbb{C}^{+} \mid \operatorname{Re}(z)<0\right\}\right) \subset \operatorname{cl} \mathbb{C}^{-},
$$

where $\mathbb{C}^{-}$denotes the set $\mathbb{C}^{-}=\{z \in \mathbb{C} \mid \operatorname{Im}(z)<0\}$. To prove that $u$ is a Stieltjes function positive on $\mathbb{R}^{+*}$, it just remains to show that $u(x)>0$ for $x>0$. By using H3, we immediately get that $u(x)=f\left(\mathrm{i} x^{\frac{1}{2}}\right) \in \mathbb{R}$, for $x>0$. Then, the positivity of $u(x)$ follows from the decreasing nature of the real function $y \mapsto f(\mathrm{i} y), y \in \mathbb{R}^{+}$which implies, by virtue of H2, that $f(i y) \geq$ $\lim _{y \rightarrow \infty} f(\mathrm{i} y)=f_{\infty}>0$. This decreasing property is an immediate consequence of the CauchyRiemann relations written on the positive imaginary axis: $\partial_{y} \operatorname{Re} f(0, y)=-\partial_{x} \operatorname{Im} f(0, y)$ and the fact that $\partial_{x} \operatorname{Im} f(0, y) \geq 0$ by Lemma 8 .

Figure 1 sums up the effect of the square root mapping applied to the function $f$ to convert it into the Stieltjes function $u$, defined by (2.15).

Corollary 10. The function $v$ defined by

$$
v(z):=z u(-z)=z f(\sqrt{z}), \forall z \in \mathbb{C}
$$

is a Herglotz function, which is analytic on $\mathbb{C} \backslash \mathbb{R}^{+}$and negative on $\mathbb{R}^{-*}$. Moreover, in its representation given by Theorem 2 , the measure $\mathrm{m}$ is supported in $\mathbb{R}^{+}$and $\alpha$ is equal to $f_{\infty}$. 
Proof. The following proof is partially inspired from [9]. First, one notices from definition (2.16) and Theorem 9 that $v$ is analytic on $z \in \mathbb{C} \backslash \mathbb{R}^{+}$and negative on $\mathbb{R}^{-*}$. Then, as $u$ is defined by (2.15) is a Stieltjes function which tends to $f_{\infty}$ when $|z| \rightarrow \infty$, by the representation Theorem 6. $u$ can be expressed as

$$
u(z)=f_{\infty}+\int_{\mathbb{R}^{+}} \frac{\mathrm{d} \nu(\xi)}{\xi+z}, \forall z \in \mathbb{C} \backslash \mathbb{R}^{-},
$$

with $\nu$ a positive regular Borel measure on $\mathbb{R}^{+}$such that $\int_{\mathbb{R}^{+}} \mathrm{d} \nu(\xi) /(1+\xi)$ is finite. Thus, the function $v$ defined by (2.16) is given by

$$
v(z)=f_{\infty} z+\int_{\mathbb{R}_{+}} \frac{z \mathrm{~d} \nu(\xi)}{\xi-z}, \forall z \in \mathbb{C} \backslash \mathbb{R}^{+},
$$

and therefore

$$
\operatorname{Im} v(z)=f_{\infty} \operatorname{Im}(z)+\int_{\mathbb{R}_{+}} \frac{\xi \operatorname{Im}(z)}{|\xi-z|^{2}} \mathrm{~d} \nu(\xi)>0, \text { when } \operatorname{Im}(z)>0 .
$$

Hence, one concludes that $v$ is a Herglotz function. Furthermore, from the definition (2.16) of $v$ and the hypothesis $\mathrm{H} 2$, one gets immediately that its coefficient $\alpha$ in the representation Theorem 6 is equal to $f_{\infty}$. Finally, as $v$ is analytic on $z \in \mathbb{C} \backslash \mathbb{R}^{+}$and negative on $\mathbb{R}^{-*}$, one deduces from 2.10 that the support of the measure $m$ associated with $v$ is included in $\mathbb{R}^{+}$.

Remark 11. The assumption H1 supposes that $f$ can be continuously extended from the upperhalf plane to the real line and implies in particular that $f$ admits no poles on the real axis or equivalently (see [23]) that the measure $\nu$ associated with the Herglotz function $v$ has no punctual part. Indeed, we can relax this hypothesis by considering functions $f$ of the form:

$f(z)=f_{c}(z)+f_{p}(z)$ with $f_{p}(z)=-\sum_{n=1}^{N} \frac{A_{n}}{z^{2}-\xi_{n}}$ for $N \in \mathbb{N}, A_{1}, \cdots, A_{N}>0$ and $\xi_{1}, \cdots, \xi_{N} \geq 0$,

where $f_{c}$ satisfies the hypotheses H1-4. Then, it is straightforward to check that Theorem 2.16 and Corollary 10 still hold (except that the definitions (2.15) and (2.16) of $u$ and $v$ do not hold on the poles of $f$ ). In electromagnetism, the function $f$ can be seen as the dielectric permittivity $\varepsilon$ or the magnetic permeability $\mu$ as a function of the frequency $z=\omega$. In this context, functions $f=f_{\infty}+f_{p}$ correspond to the constitutive laws of non-dissipative generalized Lorentz models for which $\varepsilon$ or $\mu$ are rational functions of the frequency with real coefficients (see [89, 88, 17]).

Remark 12. In the literature [18, 12, 90], one finds also another Herglotz function constructed from functions $f$ satisfying the hypothesis H1-4, namely

$$
\tilde{v}(z)=z f(z), \forall z \in \operatorname{cl} \mathbb{C}^{+} .
$$

Indeed, to prove that the imaginary part of $\tilde{v}$ is non-negative for $z \in \mathbb{C}^{+}$, one follows the same arguments as in the proof of Lemma 8 by applying the maximum principle to the function $\operatorname{Im}\left(z\left(f-f_{\infty}\right)\right)$ on $\mathrm{cl} \mathbb{C}^{+}$. Nevertheless, instead of H2, this requires a more stringent decreasing assumption at infinity: $f(z)=f_{\infty}+o(1 / z)$, with $f_{\infty}>0$ when $|z| \rightarrow \infty$ in $\mathrm{cl}^{+}$. 
Unlike $v, \tilde{v}$ does not derive from a Stieltjes function, thus it does not satisfy the additional properties that it has an analytic extension in $\mathbb{C} \backslash \mathbb{R}^{+}$which is negative on $\mathbb{R}^{-*}$. Therefore, the measure $\mathrm{m}$ associated with $\tilde{v}$ in Theorem 2 is not necessarily supported in $\mathbb{R}^{+}$. Nevertheless, $\tilde{v}$ has the advantage to satisfy the additional relation:

$$
h(z)=-\overline{h(-\bar{z})}, \forall z \in \mathrm{cl} \mathbb{C}^{+}
$$

(which can be deduced from H3). One will see in the following that using $\tilde{v}$ instead of $v$ will lead to slightly different bounds on the function $f$.

\subsection{General bounds on the function $f$}

Our aim is now to derive bounds on a function $f$ which satisfies the hypotheses H1-4 on a finite interval $\left[x_{-}, x_{+}\right] \subset \mathbb{R}^{+*}$. The key step is to use the analytic properties of its associated Herglotz function $v$ defined by Corollary 10 which relies on the existence of the Stieltjes function $u$ of Theorem 9. To this end, we follow the approach of [31, 12] by using the sum rules integral identities established in [12], recalled here in Proposition 4. Our resulting bounds generalize the ones developed in [31, 12. Moreover, they are optimal in the sense that they maximize the sum rules $(2.12)$ over the finite interval $\left[x_{-}, x_{+}\right] \subset \mathbb{R}^{+, *}$ in the sense of Theorem 14 .

Let $\Delta>0$, we denote by $h_{\mathrm{m}}$ the Herglotz function defined by:

$$
h_{\mathrm{m}}(z)=\int_{-\Delta}^{\Delta} \frac{\operatorname{dm}(\xi)}{\xi-z}, \forall z \in \mathbb{C}^{+},
$$

where $\mathrm{m} \in \mathcal{M}_{\Delta}$. Here $\mathcal{M}_{\Delta}$ stands for the set finite regular positive Borel measure $\mathrm{m}$ whose support is included in the interval $[-\Delta, \Delta]$ and whose total mass is normalized to 1 , in other words: $\mathrm{m}(\mathbb{R})=\mathrm{m}([-\Delta, \Delta])=1$, for all $\mathrm{m} \in \mathcal{M}_{\Delta}$.

Our goal is to derive bounds on $f$ by using the sum rule 2.12 on the function $v_{\mathrm{m}}$ :

$$
v_{\mathrm{m}}(z)=h_{\mathrm{m}}(v(z)) \text { on } \mathbb{C}^{+}
$$

where $v$ is the Herglotz function defined via $f$ in Corollary 10. As $v$ is not constant, one first notices that $v_{\mathrm{m}}$ is a Herglotz function as it is a composition of two Herglotz functions (see [9]). To apply the sum rules, we need the asymptotic behavior of $v_{\mathrm{m}}$ near zero and infinity. It is the purpose of the following lemma.

Lemma 13. For any $\theta \in\left(0, \frac{\pi}{2}\right)$, the Herglotz function $h_{\mathrm{m}}$ satisfies the following asymptotics in the Stolz domain $D_{\theta}$ :

$$
h_{\mathrm{m}}(z)=-\frac{\mathrm{m}(\{0\})}{z}+o\left(\frac{1}{z}\right) \quad \text { as }|z| \rightarrow 0 \text { and } h_{\mathrm{m}}(z)=-\frac{1}{z}+o\left(\frac{1}{z}\right) \text { as }|z| \rightarrow+\infty,
$$

which imply that in $D_{\theta}$ :

$$
v_{\mathrm{m}}(z)=-\frac{\mathrm{m}(\{0\})}{f(0) z}+o\left(\frac{1}{z}\right) \quad \text { as }|z| \rightarrow 0 \quad \text { and } \quad v_{\mathrm{m}}(z)=-\frac{1}{f_{\infty} z}+o\left(\frac{1}{z}\right) \quad \text { as }|z| \rightarrow+\infty .
$$


Proof. The asymptotic behavior at $z=0$ of $h_{\mathrm{m}}$ follows from the relation (2.11) which is proved in [11] by using Lebesgue's dominated convergence theorem. To show the asymptotics (2.21) of $h$ at $z=\infty$, one gets first, using the relation (2.20), that:

$$
z h_{\mathrm{m}}(z)=\int_{-\Delta}^{\Delta} \frac{z \mathrm{dm}(\xi)}{\xi-z}=-\mathrm{m}([-\Delta, \Delta])+\int_{-\Delta}^{\Delta} \frac{\xi \mathrm{dm}(\xi)}{\xi-z},
$$

where $\mathrm{m}([-\Delta, \Delta])=1$, by hypothesis. One finally concludes by proving that the integral of the right hand side in the latter expression tends to 0 as $|z| \rightarrow+\infty$. This is a consequence of Lebesgue's dominated convergence theorem where a domination condition on the integrand is given by

$$
\left|\frac{\xi}{\xi-z}\right| \leq \frac{1}{\sin (\theta)}, \quad \text { since }|\xi-z| \geq|\xi| \sin (\theta), \forall \xi \in[-\Delta, \Delta] \text { and } \forall z \in D_{\theta} \text {. }
$$

The asymptotics 2.22 follows immediately by composition from the asymptotics (2.21) and the hypotheses $\mathrm{H} 1$ and $\mathrm{H} 2$ which imply respectively that in $D_{\theta}: v(z)=f(0) z+o(z)$, as $|z| \rightarrow 0$ and $v(z)=f_{\infty} z+o(z)$, as $|z| \rightarrow+\infty$ (one notices that the first asymptotic formula in (2.22) is well-defined. Indeed $f(0)$ is positive since we already showed (see proof of Theorem 9 that the function $f(z)$ is real and decreasing along the imaginary axis, thus $\left.f(0) \geq f_{\infty}>0\right)$.

One can now use the sum rules 2.12 on the function $v_{\mathrm{m}}$ over the finite frequency band $\left[x_{-}, x_{+}\right]$to get the following inequality:

$$
\lim _{y \rightarrow 0^{+}} \frac{1}{\pi} \int_{x_{-}}^{x_{+}} \operatorname{Im} v_{\mathrm{m}}(x+\mathrm{i} y) \mathrm{d} x \leq \frac{1}{f_{\infty}}-\frac{\mathrm{m}(\{0\})}{f(0)} \leq \frac{1}{f_{\infty}}
$$

where the right inequality in the latter expression is justified by the fact that $\mathrm{m}$ is a positive measure and that $f(0)$ is also positive.

The following theorem expresses that if one wants to maximize the sum rules 2.23 ) on the set of measures $\mathcal{M}_{\Delta}$, it is sufficient to use Dirac measures: $\mathrm{m}=\delta_{\xi}$ for points $\xi \in[-\Delta,+\Delta]$.

Theorem 14. Let $\Delta$ be a positive real number and $\left[x_{-}, x_{+}\right]$be a finite frequency band included in $\mathbb{R}^{+*}$, then one has

$$
\sup _{\mathrm{m} \in \mathcal{M}_{\Delta}} \frac{1}{\pi} \lim _{y \rightarrow 0^{+}} \int_{x_{-}}^{x_{+}} \operatorname{Im} v_{\mathrm{m}}(x+\mathrm{i} y) \mathrm{d} x=\sup _{\xi \in[-\Delta,+\Delta]} \frac{1}{\pi} \lim _{y \rightarrow 0^{+}} \int_{x_{-}}^{x_{+}} \operatorname{Im} v_{\delta_{\xi}}(x+\mathrm{i} y) \mathrm{d} x .
$$

Proof. Let $\mathrm{m} \in \mathcal{M}_{\Delta}$, one denotes by $\nu_{\mathrm{m}}$ the measure associated with the Herglotz function: $v_{\mathrm{m}}$ by the representation Theorem 2. Thus, by virtue of the relation 2.10 which defines the measure of a Herglotz function, one has:

$$
\frac{1}{\pi} \lim _{y \rightarrow 0^{+}} \int_{x_{-}}^{x_{+}} \operatorname{Im} v_{\mathrm{m}}(x+\mathrm{i} y) \mathrm{d} x=\frac{\nu_{\mathrm{m}}\left(\left(x_{-}, x_{+}\right)\right)+\nu_{\mathrm{m}}\left(\left[x_{-}, x_{+}\right]\right)}{2} .
$$

One wants now to connect the measure $\nu_{\mathrm{m}}$ of $v_{\mathrm{m}}=h_{\mathrm{m}} \circ v$ to the measure $\mathrm{m}$ of the Herglotz function $h_{\mathrm{m}}$. In [19], the authors provide an expression of the measure $\nu_{\mathrm{m}}$ in terms of the 
measures $\mathrm{m}$ and the measure $\nu_{\delta_{\xi}}$ associated with the Herglotz function $v_{\delta_{\xi}}=h_{\delta_{\xi}} \circ v$. They prove that for any Borelian sets $B, \nu_{\mathrm{m}}(B)$ is given by

$$
\nu_{\mathrm{m}}(B)=\int_{-\Delta}^{\Delta} \nu_{\delta_{\xi}}(B) \mathrm{dm}(\xi)
$$

Thus, applying this last relation to $B=\left(x_{-}, x_{+}\right)$and $B=\left[x_{-}, x_{+}\right]$in the equation 2.25) yields:

$$
\begin{aligned}
\frac{1}{\pi} \lim _{y \rightarrow 0^{+}} \int_{x_{-}}^{x_{+}} \operatorname{Im} v_{\mathrm{m}}(x+\mathrm{i} y) \mathrm{d} x & =\int_{-\Delta}^{\Delta} \frac{1}{2}\left[\nu_{\delta_{\xi}}\left(\left(x_{-}, x_{+}\right)\right)+\nu_{\delta_{\xi}}\left(\left[x_{-}, x_{+}\right]\right)\right] \mathrm{dm}(\xi), \\
& \leq \sup _{\xi \in[-\Delta,+\Delta]}\left(\frac{1}{2}\left[\nu_{\delta_{\xi}}\left(\left(x_{-}, x_{+}\right)\right)+\nu_{\delta_{\xi}}\left(\left[x_{-}, x_{+}\right]\right)\right]\right) \mathrm{m}([-\Delta,+\Delta]) .
\end{aligned}
$$

Using now the fact that $\mathrm{m}([-\Delta, \Delta])=1$ and the relation 2.10 which characterizes the measure $\nu_{\delta_{\xi}}$ of the Herglotz function $v_{\delta_{\xi}}$ lead us to:

$$
\frac{1}{\pi} \lim _{y \rightarrow 0^{+}} \int_{x_{-}}^{x_{+}} \operatorname{Im} v_{\mathrm{m}}(x+\mathrm{i} y) \mathrm{d} x \leq \sup _{\xi \in[-\Delta,+\Delta]} \frac{1}{\pi} \lim _{y \rightarrow 0^{+}} \int_{x_{-}}^{x_{+}} \operatorname{Im} v_{\delta_{\xi}}(x+\mathrm{i} y) \mathrm{d} x .
$$

By taking the supremum on $\mathcal{M}_{\Delta}$, one shows one side of the equality (2.24). As the reverse inequality of (2.24) is straightforward, this concludes the proof.

The last theorem shows that for any $\Delta \in \mathbb{R}$, the family of Dirac measures $\left(\delta_{\xi}\right)_{\xi \in \mathbb{R}}$ maximizes the sum rule 2.23) on the set of probability measures $\mathcal{M}_{\Delta}$. For such measures, the inequality 2.23 can be rewritten as:

$$
\lim _{y \rightarrow 0^{+}} \int_{x_{-}}^{x_{+}} \operatorname{Im} v_{\delta_{\xi}}(x+\mathrm{i} y) \mathrm{d} x=\lim _{y \rightarrow 0^{+}} \int_{x_{-}}^{x_{+}} \operatorname{Im}\left(\frac{1}{\xi-v(x+\mathrm{i} y)}\right) \mathrm{d} x \leq \frac{\pi}{f_{\infty}}, \forall \xi \in \mathbb{R} .
$$

\subsection{The case of a transparency window}

By using the family of punctual measures $\left(\delta_{\xi}\right)_{\xi \in \mathbb{R}}$, we will now derive an explicit bound on the function $f$ on a interval $\left[\omega_{-}, \omega_{+}\right] \subset \mathbb{R}^{+*}$ under the assumption that this interval is a transparency window. In other words, one supposes that $f$ is real on $\left[\omega_{-}, \omega_{+}\right]$. In physics, (like in electromagnetism when for instance $f=\varepsilon$ or $f=\mu$ ), this hypothesis amounts neglecting the absorption of the material in the frequency band $\left[\omega_{-}, \omega_{+}\right]$.

In this case, one gets immediately that the Herglotz function $v$ is real on $\left[x_{-}, x_{+}\right]=\left[\omega_{-}^{2}, \omega_{+}^{2}\right]$. Thus, one can extend $v$ analytically through the interval $\left(x_{-}, x_{+}\right)$by using Schwarz's reflection principle by posing

$$
v_{e}(z)=v(z) \text { on } \mathrm{cl} \mathbb{C}^{+} v_{e}(z)=\overline{v(\bar{z})} \text { on } \mathbb{C}^{-}
$$

and it is straightforward to check (thanks to H3) that $v_{e}$ coincides with the definition (2.16) of $v$ on the domain $D=\mathbb{C} \backslash\left(\left[0, x_{-}\right] \cup\left[x_{+},+\infty\right]\right)$. Hence, the function $v$ is analytic on $D$. With our approach, one recovers in the next proposition a bound similar to the ones derived in [56, 91]. This bound correlates the value of two points of the function $f$ within the considered interval. A generalization of such bounds to an arbitrary number of points of correlation is done in [56]. 
Proposition 15. In the transparency window $\left[x_{-}, x_{+}\right]=\left[\omega_{-}^{2}, \omega_{+}^{2}\right]$, the function $v$ satisfies

$$
f_{\infty}\left(x-x_{0}\right) \leq v(x)-v\left(x_{0}\right), \forall x, x_{0} \in\left[x_{-}, x_{+}\right] \text {such that } x_{0} \leq x,
$$

which yields the following bound on $f$ :

$$
\omega_{0}^{2}\left(f\left(\omega_{0}\right)-f_{\infty}\right) \leq \omega^{2}\left(f(\omega)-f_{\infty}\right), \forall \omega, \omega_{0} \in\left[\omega_{-}, \omega_{+}\right] \text {such that } \omega_{0} \leq \omega .
$$

Proof. Let $x_{0} \in\left(x_{-}, x_{+}\right)$. One defines $\xi$ by $\xi=v\left(x_{0}\right) \in \mathbb{R}$. Hence, the Herglotz function $v_{\delta_{\xi}}=(\xi-v)^{-1}$ has a pole at $z=x_{0}$. As any real pole of a Herglotz functions is of multiplicity one (see [23]), this implies in particular that the derivative $v^{\prime}\left(x_{0}\right) \neq 0$. Moreover, as $\xi-v$ is an analytic function which is not constant on $D$, therefore the pole $x_{0}$ is isolated. Thus, there exists a closed interval: $\left[\tilde{x}_{-}, \tilde{x}_{+}\right] \subset\left(x_{-}, x_{+}\right)$containing $x_{0}$ such that $x_{0}$ is the only singular point of the function $v_{\delta_{\xi}}$ on $\left[\tilde{x}_{-}, \tilde{x}_{+}\right]$. Hence, one can rewrite $v_{\delta_{\xi}}$ as:

$$
v_{\delta_{\xi}}(z)=\frac{g(z)}{\left(z-x_{0}\right)} \text { where } g \text { is analytic and real on }\left[\tilde{x}_{-}, \tilde{x}_{+}\right] \text {and } g\left(x_{0}\right)=\frac{-1}{v^{\prime}\left(x_{0}\right)} \text {. }
$$

Using this last property on $v_{\delta_{\xi}}$, one can evaluate the limit in the left hand side of $(2.26)$ :

$$
\begin{aligned}
& \lim _{y \rightarrow 0^{+}} \int_{\tilde{x}_{-}}^{\tilde{x}^{+}} \operatorname{Im} v_{\delta_{\xi}}(x+\mathrm{i} y) \mathrm{d} x \\
= & \lim _{y \rightarrow 0^{+}} \int_{\tilde{x}_{-}}^{\tilde{x}^{+}} \operatorname{Im}\left(\frac{g(x+\mathrm{i} y)-g\left(x_{0}\right)}{x+\mathrm{i} y-x_{0}}\right) \mathrm{d} x+\lim _{y \rightarrow 0^{+}} \int_{\tilde{x}_{-}}^{\tilde{x}^{+}} \operatorname{Im}\left(\frac{-g\left(x_{0}\right)}{x-\left(x_{0}+\mathrm{i} y\right)}\right) \mathrm{d} x .
\end{aligned}
$$

Indeed, as a consequence of Lebesgue's dominated convergence theorem, the first limit of (2.29) is 0 and by applying the Sokhotski-Plemelj formula (see [34]) to evaluate the second limit of 2.29 , one gets:

$$
\lim _{y \rightarrow 0^{+}} \int_{\tilde{x}_{-}}^{\tilde{x}^{+}} \operatorname{Im} v_{\delta_{\xi}}(x+\mathrm{i} y) \mathrm{d} x=-\pi g\left(x_{0}\right)=\frac{\pi}{v^{\prime}\left(x_{0}\right)},
$$

By using (2.26), this leads to:

$$
f_{\infty} \leq v^{\prime}\left(x_{0}\right), \forall x_{0} \in\left(x_{-}, x_{+}\right) .
$$

Integrating this latter relation leads to inequality (2.27) on $\left(x_{-}, x_{+}\right)$, which extends to the closed interval $\left[x_{-}, x_{+}\right]$by using the continuity of $v$ at $x_{ \pm}$. One finally derives inequality (2.28) from 2.27 by using the definition 2.16 of $v$ and the changes of variables: $x=\omega^{2}$ and $x_{0}=\omega_{0}^{2}$.

\section{Link with the Kramers-Kronig relations}

For the case of a transparency window: $\left[\omega_{-}, \omega_{+}\right]$, we want now to emphasize that the bound obtained in the proposition 15 can be also derived by applying the Kramers-Kronig relations to the function $f$ :

$$
\operatorname{Re} f(\omega)=f_{\infty}+\frac{2}{\pi} \mathcal{P} \int_{0}^{\infty} \frac{\omega^{\prime} \operatorname{Im} f\left(\omega^{\prime}\right)}{\left(\omega^{\prime}\right)^{2}-\omega^{2}} d \omega^{\prime}
$$

where $\mathcal{P}$ denotes the Cauchy principal value of the integral. In electromagnetism, these relations, satisfied by the permittivity $\varepsilon$ and the permeability $\mu$ (see [76, 35]), characterize the 
dispersion of a passive material by correlating the real part and the imaginary part of $\varepsilon$ and $\mu$ by nonlocal integral relations.

Mathematically, to derive the Kramers-Kronig relations pointwise at a frequency $\omega$, one supposes classically in addition to H1-4 that $\omega^{\prime} \rightarrow\left(f-f_{\infty}\right) / \omega^{\prime}$ is an integrable function at the vicinity of $\pm \infty$ and that $f$ is Hölder continuous at $\omega$. These two last conditions (see [34, 82]) ensure the existence of the Cauchy principal value in 2.30 . We point out that in the literature, one can find other mathematical hypotheses such as $f$ belongs to to Hardy space $H_{2}\left(\mathbb{C}^{+}\right)$(see Titchmarsh's theorem [76]) which ensure the existence of these relations for almost every real frequencies $\omega$.

Now using the fact $\left[\omega_{-}, \omega_{+}\right]$is a transparency window, i. e. $\operatorname{Im} f(\omega)=0$ for all $\omega \in\left[\omega_{-}, \omega_{+}\right]$, one gets:

$$
f(\omega)=f_{\infty}+\frac{2}{\pi} \int_{0}^{\omega_{-}} \frac{\omega^{\prime} \operatorname{Im} f\left(\omega^{\prime}\right)}{\left(\omega^{\prime}\right)^{2}-\omega^{2}} d \omega^{\prime}+\frac{2}{\pi} \int_{\omega^{+}}^{\infty} \frac{\omega^{\prime} \operatorname{Im} f\left(\omega^{\prime}\right)}{\left(\omega^{\prime}\right)^{2}-\omega^{2}} d \omega^{\prime}, \forall \omega \in\left(\omega_{-}, \omega_{+}\right),
$$

where the Cauchy principal value is not useful anymore in the latter expression since in both integrals the singular point does not belong to the domain of integration. Moreover, in a transparency window, the function $f$ can be analytically, by a Schwarz reflection principle, extended through the interval, thus the Hölder regulartity is satisfied on $\left(\omega_{-}, \omega_{+}\right)$.

Applying the Kramers-Kronig relation 2.31) to two frequencies $\omega, \omega_{0} \in\left(\omega_{-}, \omega_{+}\right)$satisfying $\omega_{0} \leq \omega$ yields

$$
\begin{aligned}
\omega^{2}\left[f(\omega)-f_{\infty}\right]-\omega_{0}^{2}\left[f\left(\omega_{0}\right)-f_{\infty}\right]= & \frac{2}{\pi} \int_{0}^{\omega_{-}} \omega^{\prime} \operatorname{Im} f\left(\omega^{\prime}\right)\left[\frac{\omega^{2}}{\left(\omega^{\prime}\right)^{2}-\omega^{2}}-\frac{\omega_{0}^{2}}{\left(\omega^{\prime}\right)^{2}-\omega_{0}^{2}}\right] d \omega^{\prime} \\
& +\frac{2}{\pi} \int_{\omega_{+}}^{\infty} \omega^{\prime} \operatorname{Im} f\left(\omega^{\prime}\right)\left[\frac{\omega^{2}}{\left(\omega^{\prime}\right)^{2}-\omega^{2}}-\frac{\omega_{0}^{2}}{\left(\omega^{\prime}\right)^{2}-\omega_{0}^{2}}\right] d \omega^{\prime} \\
= & \frac{2}{\pi} \int_{0}^{\omega_{-}} \omega^{\prime} \operatorname{Im} f\left(\omega^{\prime}\right)\left[\frac{\left(\omega^{\prime}\right)^{2}\left(\omega^{2}-\omega_{0}^{2}\right)}{\left[\left(\omega^{\prime}\right)^{2}-\omega^{2}\right]\left[\left(\omega^{\prime}\right)^{2}-\omega_{0}^{2}\right]}\right] d \omega^{\prime} \\
& +\frac{2}{\pi} \int_{\omega_{+}}^{\infty} \omega^{\prime} \operatorname{Im} f\left(\omega^{\prime}\right)\left[\frac{\left(\omega^{\prime}\right)^{2}\left(\omega^{2}-\omega_{0}^{2}\right)}{\left[\left(\omega^{\prime}\right)^{2}-\omega^{2}\right]\left[\left(\omega^{\prime}\right)^{2}-\omega_{0}^{2}\right]}\right] d \omega^{\prime} \\
\geq & 0,
\end{aligned}
$$

where to obtain the last inequality we have used the fact that the ratio

$$
\frac{\left(\omega^{2}-\omega_{0}^{2}\right)}{\left[\left(\omega^{\prime}\right)^{2}-\omega^{2}\right]\left[\left(\omega^{\prime}\right)^{2}-\omega_{0}^{2}\right]} \geq 0
$$

when either $\omega \geq \omega_{0}>\omega_{-} \geq \omega^{\prime}>0$ or when $\omega^{\prime} \geq \omega_{+}>\omega \geq \omega_{0}>0$ and the fact that H4 imposes that $\omega^{\prime} \operatorname{Im} f\left(\omega^{\prime}\right)$ is positive on $\mathbb{R}^{+}$. Thus, one obtains again the bound 2.28) on the open interval $\left(\omega_{-}, \omega_{+}\right)$. Finally, this bound can be extended to the closure of this interval by using the continuity of $f$ at $\omega_{ \pm}$.

\subsection{The lossy case}

The bound $(2.28)$ is only valid if $\operatorname{Im} f$ is exactly zero on $\left[\omega_{-}, \omega_{+}\right]$. When the loss of the material cannot be neglected in this frequency band, other bounds can be derived from the inequality 
2.23). By choosing for instance the uniform measure of $\mathcal{M}_{\Delta}$ :

$$
\operatorname{dm}(\xi)=\frac{\mathbf{1}_{[-\boldsymbol{\Delta}, \boldsymbol{\Delta}]}(\xi)}{2 \Delta} \mathrm{d} \xi
$$

for the Herglotz function $h_{\mathrm{m}}$, one recovers the bounds derived in [31]. More precisely, we get:

$$
h_{\mathrm{m}}(z)=\frac{1}{2 \Delta} \int_{-\Delta}^{\Delta} \frac{1}{\xi-z} \mathrm{~d} \xi=\frac{1}{2 \Delta} \log \left(\frac{z-\Delta}{z+\Delta}\right), \forall z \in \mathbb{C}^{+}
$$

where the function log is defined with the same branch cut: $\mathbb{R}^{+}$as the square root function (2.14. As:

$$
\frac{z-\Delta}{z+\Delta}=\frac{|z|^{2}-\Delta^{2}+2 \mathrm{i} \Delta \operatorname{Im}(z)}{\left|z+\Delta^{2}\right|}, \forall z \in \mathbb{C}^{+},
$$

one checks easily that $\operatorname{Im} h_{\mathrm{m}}(z)$ is bounded above by $\pi /(2 \Delta)$ and from below by:

$$
\operatorname{Im} h_{\mathrm{m}}(z) \geq \frac{\pi}{4 \Delta} H(\Delta-|z|), \forall z \in \mathbb{C}^{+},
$$

where $H$ stands here for the Heaviside function. Moreover, in the limit $y \rightarrow 0^{+}, \operatorname{Im}[h(x+i y)]$ takes the value $\pi /(2 \Delta)$ for $|x|<\Delta$ and 0 for $|x|>\Delta$.

Now, applying the relations 2.32 and 2.23 , one gets:

$$
\lim _{y \rightarrow 0^{+}} \int_{x_{-}}^{x_{+}} \operatorname{Im} v_{\mathrm{m}}(x+\mathrm{i} y) \mathrm{d} x=\frac{1}{2 \Delta} \lim _{y \rightarrow 0^{+}} \int_{x_{-}}^{x_{+}} \arg \left(\frac{v(x+\mathrm{i} y)-\Delta}{v(x+i \epsilon)+\Delta}\right) \mathrm{d} x \leq \frac{\pi}{f_{\infty}} .
$$

Hence, using the bound (2.33), one gets we obtain a less stringent but more transparent inequality:

$$
\lim _{y \rightarrow 0^{+}} \int_{x_{-}}^{x_{+}} H(\Delta-|v(x+\mathrm{i} y)|) \mathrm{d} x \leq \frac{4 \Delta}{f_{\infty}}
$$

and using Lebesgue's Dominated convergence theorem to evaluate this limit (thanks to the continuity assumption of $f$ on $\left.\left[x_{-}, x_{+}\right]\right)$we get

$$
\int_{x_{-}}^{x_{+}} H(\Delta-|v(x)|) \mathrm{d} x \leq \frac{4 \Delta}{f_{\infty}} .
$$

In a plot of $|v(x)|$ against $x$ the quantity on the left of $(2.35)$ represents the total length of the interval or intervals of $x$, between $x_{-}$and $x_{+}$, where $|v(x)|$ is less than $\Delta$. Clearly the bound implies that this total length must shrink to zero as $\Delta \rightarrow 0$. If we take

$$
\Delta=\max _{x \in\left[x_{-}, x_{+}\right]}|v(x)|
$$

then the left hand side of 2.35 equals $x_{+}-x_{-}$and

$$
\frac{1}{4}\left(x_{+}-x_{-}\right) f_{\infty} \leq \max _{x \in\left[x-, x_{+}\right]}|v(x)|
$$

One finally gets immediately from this last inequality the following bound on the function $f$. 
Proposition 16. Let $\left[\omega_{-}, \omega_{+}\right] \subset \mathbb{R}^{+*}$ then the function $f$ satisfies the following inequality:

$$
\frac{1}{4}\left(\omega_{+}^{2}-\omega_{-}^{2}\right) f_{\infty} \leq \max _{x \in\left[\omega_{-}, \omega_{+}\right]}\left|\omega^{2} f(\omega)\right|
$$

This last bound is essentially the same as the bound (1) derived in [31].

Remark 17. More precisely, one will recover exactly the bound (1) derived in [31], namely

$$
\frac{1}{2}\left(\omega_{+}-\omega_{-}\right) f_{\infty} \leq \max _{x \in\left[\omega_{-}, \omega_{+}\right]}|\omega f(\omega)|
$$

if one uses the Herglotz function $\tilde{v}$ defined by (2.18) instead of $v$ to define the function $v_{m}$. One points out that factor $1 / 2$ instead of the factor $1 / 4$ in (2.36) comes from the relation (2.19) satisfied by $\tilde{v}$ which allows one to rewrite the sum rule (2.12) as

$$
\lim _{\eta \rightarrow 0^{+}} \lim _{y \rightarrow 0^{+}} \frac{2}{\pi} \int_{\eta<x<\eta^{-1}} \operatorname{Im} h(x+\mathrm{i} y) \mathrm{d} x=a_{-1}-b_{-1} .
$$

Remark 18. Notice here that all the bounds derived in this section still hold for functions $f$ of the form (2.17) whose real poles do not belong to the interval $\left[\omega_{-}, \omega_{+}\right]$. In other words, it extends also to non-dissipative generalized Drude-Lorentz models whose resonances do not belong to the frequency range of interest. Thus, the hypothesis H1 which assumes that $f$ is continuous for all real frequencies can be relaxed.

\section{Bounds on the polarizability tensor and quasi-static cloaking}

\subsection{Formulation of the problem}

The challenging problem we address in this section is the following: is it possible to construct a passive material to cloak a dielectric inclusion on a whole frequency band $\left[\omega_{-}, \omega_{+}\right]$? Using the bounds derived in the first section, we will prove that it is not possible when one makes the quasi-static approximation of Maxwell's equations.

Let $\mathcal{O}$ be a bounded simply-connected dielectric inclusion with Lipschitz boundary and constant permittivity $\varepsilon \mathbf{I}$ satisfying $\varepsilon>\varepsilon_{0}$. We assume here in particular that $\mathcal{O}$ is made of a standard dielectric material for which one can neglect the dispersion, in other words the frequency dependence of $\varepsilon$, on the frequency range of interest $\left[\omega_{-}, \omega_{+}\right]$. To make invisible $\mathcal{O}$, one uses a passive cloak of any shape characterized by its dielectric tensor $\varepsilon(\mathbf{x}, \omega)$ which depends both on the spatial variable $\mathbf{x}$ and the frequency $\omega$. Thus, the cloak is composed of an anisotropic, dispersive and heterogeneous material. The whole device: the dielectric inclusion and the cloak is assumed to fill a bounded open set $\Omega \subset \mathbb{R}^{3}$ of characteristic size $R_{0}$, in other words $\Omega \subset B\left(0, R_{0}\right)$ where $B\left(0, R_{0}\right)$ denotes the sphere of radius $R_{0}$ centered at the origin. Finally, one supposes that the rest of the space: $\mathbb{R}^{3} \backslash \Omega$ has the same dielectric constant $\varepsilon_{0} \mathbf{I}$ as the vacuum. We emphasize that the cloak can surround the inclusion $\mathcal{O}$ (like in the figure 2) which is the case for many cloaking methods, but our results hold also for cloaking methods such as anomalous resonances [57] or complementary media [42] for which the inclusion can be outside the cloak. 


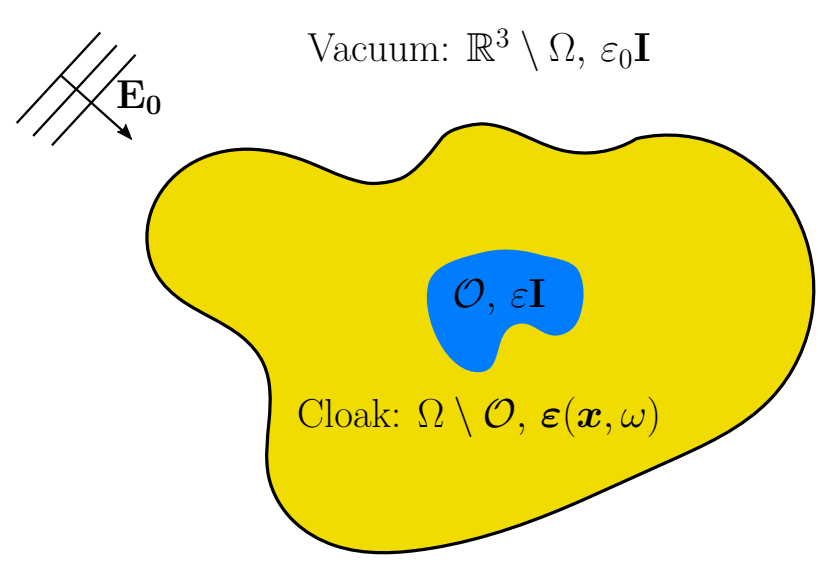

Figure 2: Description of the cloaking problem

For simplicity we assume that there is a plane incident wave on the device, with wavelength considerably larger than $R_{0}$, so that within the frequency range of interest $\omega \in\left[\omega_{-}, \omega_{+}\right]$we can use the quasi-static equations which amounts neglecting the term due to the time-derivatives of the electrical and magnetic inductions in the time-harmonic Maxwell equations. Thus, it leads to a decoupling of these equations. In this setting [35, 55, one can express the electrical field $\mathbf{E}(\mathbf{x}, \omega)$ in terms of the gradient of some potential $V(\mathbf{x}, \omega)$, i.e. $\mathbf{E}(\mathbf{x}, \omega)=-\nabla V(\mathbf{x}, \omega)$, and an incident plane wave corresponds to a uniform field $\mathbf{E}_{0} \in \mathbb{C}^{3}$ at infinity so that the potential $\nabla V(\mathbf{x}, \omega)$ has to satisfy the following elliptic equation

$$
\left\{\begin{array}{l}
\nabla \cdot(\varepsilon(\mathbf{x}, \omega) \nabla V(\mathbf{x}, \omega))=0 \text { on } \mathbb{R}^{3} \\
V(\mathbf{x}, \omega)=-\mathbf{E}_{0} \cdot \mathbf{x}+\mathcal{O}(1 /|\mathbf{x}|) \text { as }|\mathbf{x}| \rightarrow \infty .
\end{array}\right.
$$

In this context, the leading order correction to the uniform incident field $\mathbf{E}_{0}$ at infinity is a dipolar field (see [6, 35, 36, 55]), so that the potential $V$ has the asymptotic expansion

$$
V(\mathbf{x}, \omega)=-\mathbf{E}_{0} \cdot \mathbf{x}+\frac{\mathbf{p}(\omega) \cdot \mathbf{x}}{4 \pi \varepsilon_{0}|\mathbf{x}|^{3}}+\mathcal{O}\left(\frac{1}{|\mathbf{x}|^{3}}\right) \text { as }|\mathbf{x}| \rightarrow \infty
$$

where the induced dipole moment $\mathbf{p}(\omega) \in \mathbb{C}^{3}$ is linearly related to the applied field $\mathbf{E}_{0}$ and this linear relation:

$$
\mathbf{p}(\omega)=\boldsymbol{\alpha}(\omega) \mathbf{E}_{0}
$$

defines the polarizability tensor $\boldsymbol{\alpha}(\omega)$ (also called the Polya-Szego tensor), which is a $3 \times 3$ complex matrix. We point out that $\boldsymbol{\alpha}$ is a function of the frequency in the case of dispersive media. $\boldsymbol{\alpha}(\omega)$ defines the leading term of the far field of the scattered wave generated by the whole device $\Omega$. Hence, one says that the dielectric inclusion $\mathcal{O}$ is cloaked at a sufficient large distance at a frequency $\omega \in\left[\omega_{-}, \omega_{+}\right]$, if the polarizability tensor $\boldsymbol{\alpha}(\omega)$ vanishes at $\omega$.

We emphasize that the equations (3.37)-(3.39) which define the polarizability tensor $\boldsymbol{\alpha}(\omega)$ are physically relevant only in the frequency interval of interest $\left[\omega_{-}, \omega_{+}\right]$where the quasi-static approximation is valid. Nevertheless, as the dielectric tensor of the cloak $\varepsilon(\mathbf{x}, \omega)$ is defined, by 
the constitutive laws, for all frequencies $\omega$ in the closure of the upper-half plane $\mathrm{cl} \mathbb{C}^{+}$, one can study mathematically these equations for $\omega \in \mathrm{cl} \mathbb{C}^{+}$. To define their extension to $\omega \in \mathrm{cl} \mathbb{C}^{+}$, we set also that within the dielectric inclusion $\mathcal{O}$, the permittivity $\varepsilon(\cdot, \omega)$ is constant and equal to $\varepsilon \mathbf{I}$ for all $\omega \in \mathrm{cl} \mathbb{C}^{+}$. This definition is also physically relevant only in the frequency band $\left[\omega_{-}, \omega_{+}\right]$where $\mathcal{O}$ is assumed to be a non-dispersive dielectric (since the only material which behaves as a non-dispersive media at all frequencies is the vacuum). Outside the cloaking device $\Omega$, where the dielectric behaves as the vacuum, one extends $\varepsilon(\cdot, \omega)$ by $\varepsilon_{0} \mathbf{I}$ for all $\omega \in \mathrm{cl} \mathbb{C}^{+}$. To sum up, the extension of the equations (3.37)-3.39 to $\omega \in \operatorname{cl} \mathbb{C}^{+}$is performed to derive quantitative bounds on $\boldsymbol{\alpha}(\omega)$ which have a physical meaning only in the frequency band of interest $\left[\omega_{-}, \omega_{+}\right]$.

We have now to specify what we mean by a passive cloak. In the following, we equip the space of complex $3 \times 3$ matrices with the induced $l^{2}$ norm. A passive cloak is defined as a material which satisfies the following assumptions:

- $\tilde{\mathrm{H}} 1$ : for a. e. $\mathbf{x} \in \Omega \backslash \mathcal{O}, \boldsymbol{\varepsilon}(\mathbf{x}, \cdot)$ is analytic on $\mathbb{C}^{+}$and continuous on $\mathrm{cl} \mathbb{C}^{+}$,

- $\tilde{\mathrm{H}} 2$ : for a. e. $\mathbf{x} \in \Omega \backslash \mathcal{O}, \boldsymbol{\varepsilon}(\mathbf{x}, \omega) \rightarrow \varepsilon_{0} \mathbf{I}$ as $|\omega| \rightarrow \infty$ in $\mathrm{cl}^{+}$,

- $\tilde{\mathrm{H}} 3$ : for a .e. $\mathbf{x} \in \Omega \backslash \mathcal{O}, \forall \omega \in \operatorname{cl} \mathbb{C}^{+}, \varepsilon(\mathbf{x},-\bar{\omega})=\overline{\varepsilon(\mathbf{x}, \omega)}$,

- $\tilde{\mathrm{H}} 4$ : for a. e. $\mathbf{x} \in \Omega \backslash \mathcal{O}, \forall \omega \in \mathbb{R}^{+}, \operatorname{Im} \varepsilon(\mathbf{x}, \omega) \geq 0$ (passivity),

- $\tilde{\mathrm{H}}$ 5: for a. e. $\mathbf{x} \in \Omega \backslash \mathcal{O}, \forall \omega \in \operatorname{cl} \mathbb{C}^{+}, \varepsilon(\mathbf{x}, \omega)^{\top}=\varepsilon(\mathbf{x}, \omega)$, where $\top$ stands for the transpose operation (reciprocity principle).

Assumptions $\tilde{\mathrm{H}} 1-4$ correspond to hypotheses H $1-4$ (given in section 2.1) but are expressed in the more general case of anisotropic and heterogeneous passive materials [18, 55, 90. The assumption $\tilde{\mathrm{H}} 5$ is classical. Physically it means that the cloak satisfies a reciprocity principle. It is shared by most of the electromagnetic media, but it can be violated in some particular cases as for gyroscopic media or in the presence of Hall effect or magnetic-optical effect [43. Most of our bounds still hold when the reciprocity principle $\tilde{\mathrm{H}} 5$ is broken, therefore in the following we specify the results for which this additional hypothesis is required.

In the following, one extends the definition of $\varepsilon$ to $\omega=\infty$ by posing:

$$
\varepsilon(\infty, \mathbf{x})=\varepsilon \mathbf{I} \text { for a. e. } \mathbf{x} \text { in } \mathcal{O} \text { and } \varepsilon(\infty, \mathbf{x})=\varepsilon_{0} \mathbf{I} \text { for a. e. } \mathbf{x} \in \mathbb{R}^{3} \backslash \mathcal{O},
$$

so that $\varepsilon(\mathbf{x}, \cdot)$ is continuous on $\mathrm{cl} \mathbb{C}^{+} \cup\{\infty\}$ for a. e $\mathbf{x} \in \mathbb{R}^{3}$ (by hypotheses $\tilde{\mathrm{H}} 1$ and $\tilde{\mathrm{H}} 2$ ).

In this context, the broadband passive problem can be rephrased as follows: is it possible

to construct a passive cloak, in other words, a material satisfying the five hypotheses $\tilde{\mathrm{H}} 1-5$ in $\Omega \backslash \mathcal{O}$ such that the polarizability tensor $\boldsymbol{\alpha}(\omega)$ associated with the whole device $\Omega$ vanishes on the whole frequency band $\left[\omega_{-}, \omega_{+}\right]$? We will answer negatively to this question and derive quantitative bounds on the function $\boldsymbol{\alpha}$ over this frequency range.

\subsection{Analyticity of the polarizability tensor}

To derive fundamental limits on the cloaking effect over the frequency band $\left[\omega_{-}, \omega_{+}\right]$, we want to apply the bounds derived in the section 2 to the polarizability tensor $\boldsymbol{\alpha}$ or more precisely to the scalar function

$$
f(\omega)=\boldsymbol{\alpha}(\omega) \mathbf{E}_{0} \cdot \overline{\mathbf{E}_{0}} .
$$


Hence, the first step is to prove that if the dielectric tensor $\varepsilon$ satisfies $\tilde{\mathrm{H}} 1-5$ then the function $f$ satisfies the hypotheses $\mathrm{H} 1-4$ that we used to derive these bounds.

To this aim, we first recall in this subsection why equations (3.37) are well-posed, in other words why they admit a unique solution $V(\cdot, \omega)$ in a classical functional framework. Moreover, we show that $V(\cdot, \omega)$ depends analytically on the frequency $\omega$ on $\mathbb{C}^{+}$and continuously on $\operatorname{cl} \mathbb{C}^{+} \cup\{\infty\}$. Then, we use this result to prove that the function $f$, defined by $(3.40)$, shares the same regularity and satisfies the assumptions $\mathrm{H} 1-4$. Finally, this allows us (using the results of subsection 2.3 to construct a Herglotz function $v$ associated with $f$.

In this perspective, we seek the potential $V(\cdot, \omega)$, that is the solution of (3.37), in the form:

$$
V(\mathbf{x}, \omega)=-\mathbf{E}_{0} \cdot \mathbf{x}+V_{s}(\mathbf{x}, \omega)
$$

where $V_{s}(\cdot, \omega)$ denotes the scattered potential due to the reflection of the uniform field $\mathbf{E}_{0}$ on the device $\Omega$. Hence by (3.37), $V_{s}$ satisfies

$$
\left\{\begin{array}{l}
\nabla \cdot\left(\varepsilon(\mathbf{x}, \omega) \nabla V_{s}(\mathbf{x}, \omega)\right)=\nabla \cdot\left(\left(\varepsilon(\mathbf{x}, \omega)-\varepsilon_{0} \mathbf{I}\right) \mathbf{E}_{0}\right) \text { on } \mathbb{R}^{3} \\
V_{s}(\mathbf{x}, \omega)=\mathcal{O}(1 /|\mathbf{x}|) \quad \text { as }|\mathbf{x}| \rightarrow \infty
\end{array}\right.
$$

In the following, we denote respectively by $B(\omega, \delta)$ and $\|\cdot\|_{\infty}$ the open ball of center $\omega$ and radius $\delta$ and the uniform norm on $3 \times 3$ matrix valued functions defined on the set $\mathbb{R}^{3}$. We assume that the dielectric tensor $\varepsilon(\cdot, \omega)$ satisfies two additional hypotheses in the cloak $\Omega \backslash \mathcal{O}$ :

- $\tilde{\mathrm{H}} 6$ (Uniformly bounded): $\forall \omega \in \operatorname{cl} \mathbb{C}^{+}, \varepsilon(\cdot, \omega)$ is a $L^{\infty}$ matrix-valued function on $\Omega \backslash \mathcal{O}$ and it exists a positive constant $c_{1}$ such that $\sup _{\omega \mathrm{cl}} \mathbb{C}^{+}\|\varepsilon(\cdot, \omega)\|_{\infty} \leq c_{1}$,

- $\tilde{\mathrm{H}} 7$ (Coercivity):

- $\forall \omega \in \mathbb{C}^{+}$, it exists $c_{2}(\omega)>0$ and $\gamma(\omega) \in[0,2 \pi($ such that

$$
\begin{gathered}
\left|\operatorname{Im}\left(e^{i \gamma(\omega)} \varepsilon(\mathbf{x}, \omega) \mathbf{E} . \overline{\mathbf{E}}\right)\right| \geq c_{2}(\omega)|\mathbf{E}|^{2}, \quad \forall \mathbf{E} \in \mathbb{C}^{3}, \text { for a.e. } \mathbf{x} \in \mathbb{R}^{3} . \\
-\forall \omega_{0} \in \mathbb{R}, \exists \delta>0, c_{2}\left(\omega_{0}\right)>0 \text { and } \gamma\left(\omega_{0}\right) \in\left[0,2 \pi\left(\text { such that } \forall \omega \in B\left(\omega_{0}, \delta\right) \cap \operatorname{cl} \mathbb{C}^{+}:\right.\right. \\
\left|\operatorname{Im}\left(e^{i \gamma\left(\omega_{0}\right)} \varepsilon(\mathbf{x}, \omega) \mathbf{E} . \overline{\mathbf{E}}\right)\right| \geq c_{2}\left(\omega_{0}\right)|\mathbf{E}|^{2}, \quad \forall \mathbf{E} \in \mathbb{C}^{3}, \text { for a.e. } \mathbf{x} \in \mathbb{R}^{3} .
\end{gathered}
$$

Moreover, we suppose that this last property holds also in a neighborhood of $\omega_{0}=\infty$ by replacing in the previous relation $B\left(\omega_{0}, \delta\right)$ with $\{z \in \mathbb{C}|| z \mid>1 / \delta\}$.

These two hypotheses are classical assumptions. $\tilde{\mathrm{H}} 6$ amounts to suppose that the dielectric tensor is uniformly bounded with respect to $\mathbf{x}$ and $\omega$, and $\tilde{\mathrm{H}} 7$ that it is coercive with respect to $\mathbf{x}$. Moreover, we require in $\tilde{\mathrm{H}} 7$ the additional property that the constant of coercivity $c_{2}\left(\omega_{0}\right)$ holds locally in frequency in a neighborhood of any real frequency or of $\omega=\infty$.

Remark 19. The coercivity hypothesis $\tilde{\mathrm{H}} 7$ is a bit restrictive in the sense that it does not allow any type of passive media, as for instance, a cloak which behaves as a non-dissipative negative index metamaterial whose permittivity $\varepsilon(\cdot, \omega)$ is a negative constant function. In that particular case, $\varepsilon(\cdot, \omega)$ changes signs at the boundary of the cloak since the dielectric inclusion and the vacuum have positive permittivity and thus $\tilde{\mathrm{H}} 7$ is not satisfied. Nevertheless, this 
example neglects completely the dissipation of negative index materials which physically allows us to recover $\tilde{\mathrm{H}} 7$ even if it is small. However, we think that mathematically, the bounds we derived can be extended to sign-changing media by using mathematical methods associated with sign-changing conductivity equations (see [14, 66]) which do not require the coercivity of $\varepsilon(\cdot, \omega)$.

We now look for a solution $V_{s}(\cdot, \omega)$ of equations $(3.42)$ and $(3.43)$ in an appropriate weighted Sobolev space: a Beppo-Levi space, usually used as functional space for solutions of the conductivity equation in unbounded domains. It is defined by

$$
W_{1,-1}\left(\mathbb{R}^{3}\right)=\left\{u \in S^{\prime}\left(\mathbb{R}^{3}\right) \mid\left(1+|\mathbf{x}|^{2}\right)^{-\frac{1}{2}} u \in L^{2}\left(\mathbb{R}^{3}\right) \text { and } \nabla u \in \mathbf{L}^{2}\left(\mathbb{R}^{3}\right)\right\}
$$

where $S^{\prime}\left(\mathbb{R}^{3}\right), L^{2}\left(\mathbb{R}^{3}\right)$ and $\mathbf{L}^{2}\left(\mathbb{R}^{3}\right)$ denote respectively the space of tempered distributions and the spaces of scalar and vector-valued square-integrable functions. $W_{1,-1}\left(\mathbb{R}^{3}\right)$ is a Hilbert space (see [61]) for the norm

$$
\|u\|_{W_{1,-1}\left(\mathbb{R}^{3}\right)}=\|\nabla u\|_{\mathbf{L}^{2}\left(\mathbb{R}^{3}\right)}=\left(\int_{\mathbb{R}^{3}}|\nabla u|^{2} \mathrm{~d} \mathbf{x}\right)^{\frac{1}{2}} .
$$

First, we prove that the equation $\left(3.42\right.$ admits a unique solution in $W_{1,-1}\left(\mathbb{R}^{3}\right)$ which depends analytically on $\omega$ on $\mathbb{C}^{+}$and continuously on $\operatorname{cl} \mathbb{C}^{+} \cup\{\infty\}$. Then we will show that this solution satisfies the asymptotics $(3.43)$.

We denote by $\mathbf{f}(\cdot, \omega)$ the function

$$
\mathbf{f}(\cdot, \omega)=\left(\varepsilon(\cdot, \omega)-\varepsilon_{0} \mathbf{I}\right) \mathbf{E}_{0}
$$

which is compactly supported in $\Omega$. Hence, using the hypothesis $\tilde{\mathrm{H}} 6$, one checks easily that $\mathbf{f}(\cdot, \omega) \in \mathbf{L}^{2}\left(\mathbb{R}^{3}\right)$. By applying the Green formula, it is standard to show that solving the equation 3.42 in $W_{1,-1}\left(\mathbb{R}^{3}\right)$ is equivalent solving the following variational problem:

$$
\text { Find } V_{s}(\cdot, \omega) \in W_{1,-1}\left(\mathbb{R}^{3}\right) \text { such that } a_{\omega}\left(V_{s}(\cdot, \omega), v\right)=l_{\omega}(v), \forall v \in W_{1,-1}\left(\mathbb{R}^{3}\right),
$$

where the sesqulinear form $a_{\omega}$ and the anti-linear form $l_{\omega}$ are respectively defined by

$$
a_{\omega}(u, v)=\int_{\mathbb{R}^{3}} \varepsilon(\mathbf{x}, \omega) \nabla u(\mathbf{x}) \cdot \overline{\nabla v(\mathbf{x})} \mathrm{d} \mathbf{x} \text { and } l_{\omega}(v)=\int_{\mathbb{R}^{3}} \mathbf{f}(\mathbf{x}, \omega) \cdot \overline{\nabla v(\mathbf{x})} \mathrm{d} \mathbf{x}, \forall u, v \in W_{1,-1}\left(\mathbb{R}^{3}\right) .
$$

With the assumption $\tilde{\mathrm{H}} 6$ made on $\varepsilon(\cdot, \omega)$ and the Cauchy-Schwarz inequality, it is straightforward to show that

$$
\left|a_{\omega}(u, v)\right| \leq c_{1}\|u\|_{W_{1,-1}\left(\mathbb{R}^{3}\right)}\|v\|_{W_{1,-1}\left(\mathbb{R}^{3}\right)} \text { and }\left|l_{\omega}(v)\right| \leq\|\mathbf{f}(\cdot, \omega)\|_{\mathbf{L}^{2}\left(\mathbb{R}^{3}\right)}\|v\|_{W_{1,-1}\left(\mathbb{R}^{3}\right)} .
$$

In other words, $a_{\omega}$ and $l_{\omega}$ are continuous. We denote by $W_{1,-1}\left(\mathbb{R}^{3}\right)^{*}$ the dual space of $W_{1,-1}\left(\mathbb{R}^{3}\right)$ and by $\langle\cdot, \cdot\rangle$ the duality product between these two spaces. Classically, the continuity of $a_{\omega}$ allows us to define a continuous linear operator $\mathbb{A}(\omega)$ from $W_{1,-1}\left(\mathbb{R}^{3}\right)$ to $W_{1,-1}\left(\mathbb{R}^{3}\right)^{*}$ by posing:

$$
a_{\omega}(u, v)=\langle\mathbb{A}(\omega) u, \bar{v}\rangle \text { for all } u, v \in W_{1,-1}\left(\mathbb{R}^{3}\right) .
$$

The continuity of $l_{\omega}$ proves that $\mathbf{f}(\cdot, \omega) \in W_{1,-1}\left(\mathbb{R}^{3}\right)^{*}$ and can be rewritten as $l_{\omega}(v)=\langle\mathbf{f}(\cdot, \omega), \bar{v}\rangle$. Hence, the variational problem (3.45) is equivalent to solving the infinite dimensional system:

$$
\mathbb{A}(\omega) V_{s}(\cdot, \omega)=\mathbf{f}(\cdot, \omega) .
$$

For two Banach spaces $E$ and $F$, we denote in the following by $\mathcal{L}(E, F)$ the Banach space of bounded linear operators from $E$ to $F$ equipped with the operator norm. 
Lemma 20. At a fixed frequency $\omega \in \operatorname{cl} \mathbb{C}^{+} \cup\{\infty\}$, the operator $\mathbb{A}(\omega): W_{1,-1}\left(\mathbb{R}^{3}\right) \rightarrow W_{1,-1}\left(\mathbb{R}^{3}\right)^{*}$ is invertible. Moreover, the functions $\omega \mapsto \mathbb{A}(\omega)$ and $\omega \mapsto \mathbb{A}(\omega)^{-1}$ defined respectively from $\mathbb{C}^{+}$ to $\mathcal{L}\left(W_{1,-1}\left(\mathbb{R}^{3}\right), W_{1,-1}\left(\mathbb{R}^{3}\right)^{*}\right)$ and from $\mathbb{C}^{+}$to $\mathcal{L}\left(\left(W_{1,-1}\left(\mathbb{R}^{3}\right)\right)^{*}, W_{1,-1}\left(\mathbb{R}^{3}\right)\right)$ are analytic for the operator norm.

Proof. Let $\omega \in \operatorname{cl} \mathbb{C}^{+} \cup\{\infty\}$. Thanks to the hypothesis $\tilde{\mathrm{H}} 7$ on $\varepsilon(\mathbf{x}, \omega)$, we get that $a_{\omega}$ is a coercive sesquilinear form which satisfies

$$
\left|a_{\omega}(u, u)\right|=\left|e^{i \gamma(\omega)} a_{\omega}(u, u)\right| \geq\left|\operatorname{Im}\left(e^{i \gamma(\omega)} a_{\omega}(u, u)\right)\right| \geq c_{2}(\omega)\|u\|_{W_{1,-1}\left(\mathbb{R}^{3}\right)}^{2} .
$$

Thus, by Lax-Milgram's theorem, $\mathbb{A}(\omega): W_{1,-1}\left(\mathbb{R}^{3}\right) \rightarrow W_{1,-1}\left(\mathbb{R}^{3}\right)^{*}$ is an isomorphism.

One wants now to prove that the function $\omega \mapsto \mathbb{A}(\omega)$ is analytic on $\mathbb{C}^{+}$for the operator norm. For this purpose, it is sufficient to show its weak analyticity, in other words that $\omega \mapsto$ $\langle\mathbb{A}(\omega) u, \bar{v}\rangle$ is analytic on $\mathbb{C}^{+}$, for any fixed $u$ and $v \in W_{1,-1}\left(\mathbb{R}^{3}\right)$. As $\varepsilon(\mathbf{x}, \cdot)$ is analytic (by the hypothesis $\tilde{\mathrm{H}} 1$ ), one can check easily by applying the theorem of complex differentiation under the integral presented in [48] (using the hypothesis $\tilde{\mathrm{H}} 6$ for the domination condition required in its assumption) that $\omega \mapsto\langle\mathbb{A}(\omega) u, \bar{v}\rangle$ is analytic on $\mathbb{C}^{+}$for any fixed $u$ and $v \in W_{1,-1}\left(\mathbb{R}^{3}\right)$. As weak analyticity implies analyticity for the operator norm (see [37], Theorem 3.12 p. 152), the function $\omega \mapsto \mathbb{A}(\omega)$ is analytic. Therefore, one deduces (see [37] chapter 7 pp 365-366) that $\omega \mapsto \mathbb{A}(\omega)^{-1}$ is also analytic for the operator norm.

Theorem 21. At a fixed frequency $\omega \in \operatorname{cl} \mathbb{C}^{+} \cup\{\infty\}$, the equation (3.42) admits a unique solution $V_{s}(\cdot, \omega)$ in $W_{1,-1}\left(\mathbb{R}^{3}\right)$ defined by

$$
V_{s}(\cdot, \omega)=\mathbb{A}^{-1}(\omega) \mathbf{f}(\cdot, \omega) .
$$

Moreover, the function $\omega \mapsto \mathbf{E}_{s}(\cdot, \omega)=-\nabla V_{s}(\cdot, \omega)$ from $\operatorname{cl} \mathbb{C}^{+}$to $\mathbf{L}^{2}\left(\mathbb{R}^{3}\right)$ equipped with the $\|\cdot\|_{\mathbf{L}^{2}\left(\mathbb{R}^{3}\right)}$ norm is analytic on $\mathbb{C}^{+}$and continuous on $\mathrm{cl} \mathbb{C}^{+} \cup\{\infty\}$.

Proof. Let $\omega$ be in $\mathrm{cl} \mathbb{C}^{+} \cup\{\infty\}$. From the Lemma 20, we know that the operator $\mathbb{A}(\omega)$ is invertible. Hence, the equation $\left(3.42\right.$ admits a unique solution $(3.47)$ in $W_{1,-1}\left(\mathbb{R}^{3}\right)$ given by the inversion of the linear system (3.46).

Now, we show the analyticity of the function $\omega \mapsto \mathbf{E}_{s}(\cdot, \omega)$ on $\mathbb{C}^{+}$for the norm $\|\cdot\|_{\mathbf{L}^{2}\left(\mathbb{R}^{3}\right)}$ or equivalently that $\omega \mapsto \mathbf{E}_{s}(\cdot, \omega)=-\nabla V_{s}(\cdot, \omega)$ is analytic for the norm $\|\cdot\|_{W_{1,-1}\left(\mathbb{R}^{3}\right)}$. To achieve this aim, one uses the relation (3.47) and the fact that the function $\omega \mapsto \mathbb{A}(\omega)^{-1}$ is analytic for the operator norm (see Lemma 20). Thus, it only remains to prove that $\omega \mapsto \mathbf{f}(\cdot, \omega)$ is analytic for the norm of $\left(W_{1,-1}\left(\mathbb{R}^{3}\right)\right)^{*}$. By Theorem 1.37 p. 139 of [37], this is equivalent proving weak analyticity, in other words the analyticity of the functions $\omega \mapsto l_{\omega}(v)=\langle\mathbf{f}(\cdot, \omega), v\rangle$ for any fixed $v \in W_{1,-1}\left(\mathbb{R}^{3}\right)$. This last property is shown once again by applying the theorem of complex differentiation under the integral presented in [48] (using again the fact that $\varepsilon(\mathbf{x}, \cdot)$ is analytic by the hypothesis $\tilde{\mathrm{H}} 1$ and the hypothesis $\tilde{\mathrm{H}} 6$ to establish the domination condition required in this theorem).

Thus, it remains to prove the continuity of $\omega \mapsto \mathbf{E}_{s}(\cdot, \omega)=-\nabla V_{s}(\cdot, \omega)$ for real frequencies and for $\omega=\infty$. The reasoning here is slightly different from the one used for the analyticity in the upper half plane. The main reason is that weak continuity does not imply strong continuity.

Let $\left(\omega_{n}\right)$ be a sequence of $\mathrm{cl} \mathbb{C}^{+}$which tends to $\omega \in \mathbb{R}$. As $\mathbb{A}_{\omega_{n}} V_{s}\left(\cdot, \omega_{n}\right)=\mathbf{f}\left(\cdot, \omega_{n}\right)$ and $\mathbb{A}_{\omega} V_{s}(\cdot, \omega)=\mathbf{f}(\cdot, \omega)$ (where both operators $\mathbb{A}_{\omega_{n}}$ and $\mathbb{A}_{\omega}$ are invertible by Lemma 20), we get 
the following identity:

$$
V_{s}(\cdot, \omega)-V_{s}\left(\cdot, \omega_{n}\right)=\mathbb{A}_{\omega_{n}}^{-1}\left(\mathbb{A}_{\omega_{n}} V_{s}(\cdot, \omega)-\mathbb{A}_{\omega} V_{s}(\cdot, \omega)+\mathbf{f}(\cdot, \omega)-\mathbf{f}\left(\cdot, \omega_{n}\right)\right) .
$$

Thus, it follows immediately that:

$\left\|V_{s}\left(\cdot, \omega_{n}\right)-V_{s}(\cdot, \omega)\right\|_{W_{1,-1}\left(\mathbb{R}^{3}\right)} \leq\left\|\mathbb{A}_{\omega_{n}}^{-1}\right\|\left(\left\|\left(\mathbb{A}_{\omega_{n}}-\mathbb{A}_{\omega}\right) V_{s}(\cdot, \omega)\right\|_{W_{1,-1}\left(\mathbb{R}^{3}\right)^{*}}+\left\|\mathbf{f}(\cdot, \omega)-\mathbf{f}\left(\cdot, \omega_{n}\right)\right\|_{W_{1,-1}\left(\mathbb{R}^{3}\right)^{*}}\right)$

We show now that the right hand side of the last equation tends to zero. To this aim, one first remarks as a consequence of assumption $\tilde{\mathrm{H}} 6$ and Lax-Milgram's Theorem that $\left\|\mathbb{A}_{\omega_{n}}^{-1}\right\| \leq c_{2}(\omega)^{-1}$ for $n$ large enough. Then, for any $v \in W_{1,-1}\left(\mathbb{R}^{3}\right)$, one has, by using the Cauchy-Schwarz inequality:

$$
\left|\left\langle\left(\mathbb{A}_{\omega}-\mathbb{A}_{\omega_{n}}\right) V_{s}(\cdot, \omega), \bar{v}\right\rangle\right| \leq\left(\int_{\mathbb{R}^{3}}\left\|\varepsilon(\mathbf{x}, \omega)-\varepsilon\left(\mathbf{x}, \omega_{n}\right)\right\|^{2}\left|\nabla V_{s}(\mathbf{x}, \omega)\right|^{2} \mathrm{~d} \mathbf{x}\right)^{\frac{1}{2}}\|v\|_{W_{1,-1}\left(\mathbb{R}^{3}\right)} .
$$

Thus, using the continuity of $\varepsilon(\mathbf{x}, \cdot)$ at $\omega$ (assumption $\tilde{\mathrm{H}} 1$ ) and the hypothesis $\tilde{\mathrm{H}} 6$ for the domination condition, one proves, by applying Lebesgue's dominated convergence theorem, that the integral in the last formula tends to zero. Thus, one concludes that:

$$
\left\|\left(\mathbb{A}_{\omega_{n}}-\mathbb{A}_{\omega}\right) V_{s}(\cdot, \omega)\right\|_{W_{1,-1}\left(\mathbb{R}^{3}\right)^{*}}=\sup _{v \in W_{1,-1}\left(\mathbb{R}^{3}\right) \backslash\{0\}} \frac{\left|\left\langle\left(\mathbb{A}_{\omega}-\mathbb{A}_{\omega_{n}}\right) V_{s}(\cdot, \omega), \bar{v}\right\rangle\right|}{\|v\|_{W_{1,-1}\left(\mathbb{R}^{3}\right)}} \rightarrow 0 \text {, as } n \rightarrow+\infty .
$$

Finally, by doing the same reasoning for the term $\left\|\mathbf{f}(\cdot, \omega)-\mathbf{f}\left(\cdot, \omega_{n}\right)\right\|_{W_{1,-1}\left(\mathbb{R}^{3}\right)^{*}}$, one has:

$$
\left|\left\langle\mathbf{f}(\cdot, \omega)-\mathbf{f}\left(\cdot, \omega_{n}\right), v\right\rangle\right| \leq\left(\int_{\Omega}\left\|\varepsilon(\mathbf{x}, \omega)-\varepsilon\left(\mathbf{x}, \omega_{n}\right)\right\|^{2} \|\left. E_{0}\right|^{2} \mathrm{~d} \mathbf{x}\right)^{\frac{1}{2}}\|v\|_{W_{1,-1}\left(\mathbb{R}^{3}\right)},
$$

Then, by using once again Lebesgue's dominated convergence theorem (thanks to the assumptions $\tilde{\mathrm{H}} 1$ and $\tilde{\mathrm{H}} 6)$, one shows also that: $\left\|\mathbf{f}(\cdot, \omega)-\mathbf{f}\left(\cdot, \omega_{n}\right)\right\|_{W_{1,-1}\left(\mathbb{R}^{3}\right)^{*}} \rightarrow 0$, as $n \rightarrow+\infty$. Thus, one concludes that:

$$
\left\|V_{s}\left(\cdot, \omega_{n}\right)-V_{s}(\cdot, \omega)\right\|_{W_{1,-1}\left(\mathbb{R}^{3}\right)} \rightarrow 0, \text { as } n \rightarrow+\infty .
$$

The same proof as above holds to show the continuity at $\omega=\infty$.

Now, we state why the solution $V_{s}(\cdot, \omega)$ of (3.47) satisfies not only the equation $(3.42)$ but also admits the asymptotic expansion $(3.43)$ and more precisely that the leading term of $V_{s}(\cdot, \omega)$ at infinity is a dipolar field (see [35]). To this aim, one uses the fact that outside the cloaking device, the equation 3.42 becomes the following Laplace equation:

$$
\nabla^{2} u(\cdot, \omega)=0 \text { on } \mathbb{R}^{3} \backslash B(0, R) \text { and } u(\cdot, \omega)=V_{s}(\cdot, \omega) \text { on } \partial B(0, R)
$$

for $R>R_{0}$, where $\partial B(0, R)$ denotes the boundary of a ball $B(0, R)$ which contains and does not intersect the cloaking device $\Omega$. We point out that the trace of $V_{s}(\cdot, \omega)$ on the sphere $\partial B(0, R)$ belongs to $H^{1 / 2}(\partial B(0, R))$ since $V_{s}(\cdot, \omega)$ is locally $H^{1}$ (indeed, by using standard interior regularity results for second order elliptic equations, see for instance Theorem $2 \mathrm{p}$. 314 of [22], one can show that the trace of $V_{s}(\cdot, \omega)$ belongs to any $H^{s}(\partial B(0, R))$ for $\left.s>0\right)$. 
Therefore, the Dirichlet exterior problem (3.48) (see for example Theorem 2.5.14 of [61]) admits a unique solution in $W_{1,-1}\left(\mathbb{R}^{3} \backslash B(0, R)\right)$ given by the restriction of $V_{s}(\cdot, \omega)$ on $\mathbb{R}^{3} \backslash B(0, R)$ (the definition of the space $W_{1,-1}\left(\mathbb{R}^{3} \backslash B(0, R)\right)$ is deduced from the definition (3.44) by replacing $\mathbb{R}^{3}$ by $\left.\mathbb{R}^{3} \backslash B(0, R)\right)$. Moreover, as a solution of the Laplace equation 3.48 this solution admits the following integral representation:

$$
V_{s}(\mathbf{x}, \omega)=\int_{\partial B(0, R)} \frac{\partial G(\mathbf{x}, \mathbf{y})}{\partial \mathbf{n}_{y}} V_{s}(\mathbf{y}, \omega)-G(\mathbf{x}, \mathbf{y}) \frac{\partial V_{s}(\mathbf{y}, \omega)}{\partial \mathbf{n}_{y}} \text { dy with } G(\mathbf{x}, \mathbf{y})=\frac{1}{4 \pi|\mathbf{x}-\mathbf{y}|},
$$

where $\mathbf{n}_{y}=\mathbf{y} /|\mathbf{y}|$ is the outward normal of the domain $B(0, R)$. Then, by using the asymptotic expansions of the Green function $G(\mathbf{x}, \mathbf{y})$ and its normal derivative $\partial G(\mathbf{x}, \mathbf{y}) / \partial \mathbf{n}_{y}$ for large values of $|\mathbf{x}|$ :

$$
G(\mathbf{x}, \mathbf{y})=\frac{1}{4 \pi|\mathbf{x}|}+\frac{\mathbf{y} \cdot \mathbf{x}}{4 \pi|\mathbf{x}|^{3}}+O\left(\frac{1}{|\mathbf{x}|^{3}}\right) \text { and } \frac{\partial G(\mathbf{x}, \mathbf{y})}{\partial \mathbf{n}_{y}}=\frac{\mathbf{n}_{y} \cdot \mathbf{x}}{4 \pi|\mathbf{x}|^{3}}+O\left(\frac{1}{|\mathbf{x}|^{3}}\right)
$$

which holds uniformly in $\mathbf{y}$ on $\partial B(0, R)$, this leads to

$$
V_{s}(\mathbf{x}, \omega)=\frac{Q(\omega)}{4 \pi \varepsilon_{0}|\mathbf{x}|}+\frac{\mathbf{p}(\omega) \cdot \mathbf{x}}{4 \pi \varepsilon_{0}|\mathbf{x}|^{3}}+\mathcal{O}\left(\frac{1}{|\mathbf{x}|^{3}}\right),
$$

where the charge (also called monopole term) $Q(\omega)$ and the induced dipole moment $\mathbf{p}(\omega)$ are respectively given by

$$
Q(\omega)=\varepsilon_{0} \int_{\partial B(0, R)} \frac{-\partial V_{s}(\mathbf{y}, \omega)}{\partial \mathbf{n}_{y}} \mathrm{~d} \mathbf{y} \text { and } \mathbf{p}(\omega)=\varepsilon_{0} \int_{\partial B(0, R)} \frac{-\partial V_{s}(\mathbf{y}, \omega)}{\partial \mathbf{n}_{y}} \mathbf{y}+V_{s}(\mathbf{y}, \omega) \mathbf{n}_{y} \mathrm{~d} \mathbf{y} .
$$

In our scattering problem, one can easily prove that the monopole term $Q(\omega)$ vanishes. Indeed, using the divergence theorem and the equation 3.42 , one gets:

$$
\int_{\partial B(0, R)} \varepsilon(\mathbf{y}, \omega) \frac{\partial V(\mathbf{y}, \omega)}{\partial \mathbf{n}_{y}} \mathrm{~d} \mathbf{y}=\int_{\partial B(0, R)} \varepsilon_{0} \frac{\partial V(\mathbf{y}, \omega)}{\partial \mathbf{n}_{y}} \mathrm{~d} \mathbf{y}=0
$$

and thus by virtue of (3.41) and (3.50), one obtains that

$$
Q(\omega)=-\int_{\partial B(0, R)} \varepsilon_{0} \mathbf{E}_{0} \cdot \mathbf{n} \mathrm{d} \mathbf{y}=-\varepsilon_{0} \mathbf{E}_{0} \cdot \int_{\partial B(0, R)} \mathbf{n} \mathrm{d} \mathbf{y}=0 .
$$

Hence, the leading term of the scattered field $(3.49)$ is a dipolar term and using $(3.42)$, one finally gets a justification of the asymptotic formula (3.38) for the potential $V$. Our aim is now to derive a more explicit expression for the induced dipole moment $\mathbf{p}(\omega)$ by a small computation done in [55] that we reproduce here for readability. Let $\mathbf{E}_{0}$ be any vector of $\mathbb{C}^{3}$. Then, from relations (3.41), (3.42), (3.50) and the Green identity, one has:

$$
\begin{aligned}
\mathbf{p}(\omega) \cdot \overline{\mathbf{E}_{0}} & =\int_{\partial B(0, R)}-\varepsilon_{0} \frac{\partial V_{s}(\mathbf{y}, \omega)}{\partial \mathbf{n}_{y}} \mathbf{y} \cdot \overline{\mathbf{E}_{0}}+\varepsilon_{0} V_{s}(\mathbf{y}, \omega) \mathbf{n}_{y} \cdot \overline{\mathbf{E}_{0}} \mathrm{~d} \mathbf{y} \\
& =\int_{\partial B(0, R)}\left(-\varepsilon_{0} \frac{\partial V(\mathbf{y}, \omega)}{\partial \mathbf{n}_{y}}\right)\left(\mathbf{y} \cdot \overline{\mathbf{E}_{0}}\right)+\varepsilon_{0} V(\mathbf{y}, \omega) \mathbf{n}_{y} \cdot \overline{\mathbf{E}_{0}} \mathrm{~d} \mathbf{y} \\
& =\int_{B(0, R)} \varepsilon(\mathbf{y}, \omega) \mathbf{E}(\mathbf{y}, \omega) \cdot \nabla\left(\mathbf{y} \cdot \overline{\mathbf{E}_{0}}\right) \mathrm{d} \mathbf{y}-\left(\int_{B(0, R)} \varepsilon_{0} \mathbf{E}(\mathbf{y}, \omega) \mathrm{d} \mathbf{y}\right) \cdot \overline{\mathbf{E}_{0}} \\
& =\int_{B(0, R)}\left(\varepsilon(\mathbf{y}, \omega)-\varepsilon_{0} \mathbf{I}\right) \mathbf{E}(\mathbf{y}, \omega) \mathrm{d} \mathbf{y} \cdot \overline{\mathbf{E}_{0}} .
\end{aligned}
$$


As the function $\varepsilon(\cdot, \omega)-\varepsilon_{0} \mathbf{I}$ has a support contained in the cloaking device, this yields:

$$
\mathbf{p}(\omega)=\boldsymbol{\alpha}(\omega) \mathbf{E}_{0}=\int_{\Omega}\left(\varepsilon(\mathbf{y}, \omega)-\varepsilon_{0} \mathbf{I}\right) \mathbf{E}(\mathbf{y}, \omega) \mathrm{d} \mathbf{y}, \forall \omega \in \operatorname{cl} \mathbb{C}^{+} \cup\{\infty\} .
$$

Now, using this last formula, one can rewrite the function $f$ defined by $(3.40)$ as

$$
f(\omega)=\boldsymbol{\alpha}(\omega) \mathbf{E}_{0} \cdot \overline{\mathbf{E}_{0}}=\int_{\Omega}\left(\varepsilon(\mathbf{x}, \omega)-\varepsilon_{0} \mathbf{I}\right) \mathbf{E}(\mathbf{x}, \omega) \cdot \overline{\mathbf{E}_{0}} \mathrm{~d} \mathbf{x}, \forall \omega \in \operatorname{cl} \mathbb{C}^{+} \cup\{\infty\}
$$

and study its regularity with respect to the frequency $\omega$.

Proposition 22. For any fixed incident field $\mathbf{E}_{0} \in \mathbb{C}^{3}$, the function $f$ defined by (3.40) is analytic on $\mathbb{C}^{+}$and continuous on $\mathrm{cl} \mathbb{C}^{+}$, in other words, it satisfies the hypothesis $\mathrm{H} 1$.

Proof. Let $\omega \in \mathbb{C}^{+}$and $\mathbf{E}_{0}$ be a fixed vector of $\mathbb{C}^{3}$. One introduces the linear form:

$$
L_{\omega}(\mathbf{U})=\int_{\Omega}\left(\varepsilon(\mathbf{x}, \omega)-\varepsilon_{0} \mathbf{I}\right) \mathbf{U} \cdot \overline{\mathbf{E}_{0}} \mathrm{~d} \mathbf{x}, \forall \mathbf{U} \in L^{2}(\Omega)
$$

such that $f(\omega)=L_{\omega}(\mathbf{E}(\cdot, \omega))$. One easily checks that $L_{\omega}$ is well-defined and continuous by virtue of $\tilde{\mathrm{H}} 6$. Moreover, using again the theorem of complex differentiation under the integral presented in [48] (and both the hypotheses $\tilde{\mathrm{H}} 1$ and $\tilde{\mathrm{H}} 6$ to prove respectively the regularity and the domination condition required in the assumptions of this theorem), one shows that $L_{\omega}$ is weakly analytic, in other words, for any fixed $\mathbf{U} \in L^{2}(\Omega), \omega \rightarrow L_{\omega}(\mathbf{U})$ is analytic on $\mathbb{C}^{+}$. Hence, as weak analyticity implies strong analyticity (see [37, Theorem $1.37 \mathrm{p} .139$ ), $\omega \rightarrow L_{\omega}$ is also analytic. Then, using Theorem 21, one has $\omega \rightarrow \mathbf{E}(\cdot, \omega)=\mathbf{E}_{0}+\mathbf{E}_{s}(\cdot, \omega)$ is strongly analytic on $\mathbb{C}^{+}$for the $L^{2}(\Omega)$ norm and one finally deduces that $\omega \rightarrow f(\omega)=L_{\omega}(\mathbf{E}(\cdot, \omega))$ is analytic on $\mathbb{C}^{+}$.

It remains to prove the continuity of $f$ for a real frequency $\omega$. Let $\left(\omega_{n}\right)$ be a sequence of in $\operatorname{cl} \mathbb{C}^{+}$which tends to $\omega \in \mathbb{R}$. One has:

$$
\left|f\left(\omega_{n}\right)-f(\omega)\right| \leq\left|L_{\omega_{n}}\left(\mathbf{E}\left(\cdot, \omega_{n}\right)-\mathbf{E}(\cdot, \omega)\right)\right|+\left|L_{\omega_{n}}(\mathbf{E}(\cdot, \omega))-L_{\omega}(\mathbf{E}(\cdot, \omega))\right| .
$$

By $\tilde{\mathrm{H}} 6$, one checks easily that the linear form $L_{\omega}$ is uniformly bounded with respect to the frequency: $\left\|L_{\omega_{n}}\right\| \leq\left(c_{1}+\varepsilon_{0} \mid\right)\left|\mathbf{E}_{0}\right| \operatorname{mes}(\Omega)^{1 / 2}$ and by Theorem $21, \omega \rightarrow \mathbf{E}(\cdot, \omega)$ is continuous on $\operatorname{cl} \mathbb{C}^{+}$for the $L^{2}(\Omega)$ norm, thus the first term of the right hand side tends to 0 . Concerning the second term, by using hypotheses $\tilde{\mathrm{H}} 1$ and $\tilde{\mathrm{H}} 6$, it is straightforward to check , by applying Lebesgue's dominated convergence theorem, that it tends to 0 . This concludes the proof.

Remark 23. From the analyticity of the function $f$ on $\mathbb{C}^{+}$for any fixed incident field $\mathbf{E}_{0} \in \mathbb{C}^{3}$, one deduces since weak analyticity implies analyticity for the operator norm (see [37], Theorem $3.12 p$. 152) that the polarizability tensor $\boldsymbol{\alpha}$ is an analytic function of the frequency on $\mathbb{C}^{+}$with respect to the induced $l^{2}-$ norm.

We prove in the following proposition that $f$ satisfies the hypotheses H2 and H3.

Proposition 24. For any fixed non-zero incident field $\mathbf{E}_{0} \in \mathbb{C}^{3}$, the function $f$ defined by (3.40) satisfies

$$
f(\omega) \rightarrow f(\infty)=\alpha(\infty) \mathbf{E}_{0} \cdot \overline{\mathbf{E}_{0}}>0,
$$


where $\alpha(\infty)$ is defined by relation (3.52) evaluated at $\omega=\infty$. Thus, $f$ satisfies $\mathrm{H} 2$. Moreover, $f$ satisfies the hypothesis $\mathrm{H} 3$, that is

$$
f(-\bar{\omega})=\overline{f(\omega)}, \forall \omega \in \operatorname{cl} \mathbb{C}^{+} .
$$

When the cloak contains a non reciprocal medium (in other words if $\tilde{\mathrm{H}} 5$ is not satisfied), the relation (3.53) holds only under the additional assumption that $\mathbf{E}_{0}$ is a real-valued incident field.

Proof. Let $\mathbf{E}_{0}$ be a fixed vector of $\mathbb{C}^{3}$. The fact that $f(\omega) \rightarrow f(\infty)$ amounts to proving the continuity of $f$ at $\omega=\infty$, which can be dealt with in the same way as the continuity of $f$ for a real frequency $\omega$ in the proof of Proposition 22. The positivity of the limit $f(\infty)$ which has to be proved for any $\mathbf{E}_{0} \in \mathbb{C}^{3}$ turns out to be equivalent to showing that $\alpha(\infty)$ is positive-definite. This last result is a well-known property (see Theorem 4.11 of [6]) of the polarizability tensor associated with a homogeneous simple connected isotropic inclusion $\mathcal{O}$ with Lipschitz boundary embedded in the vacuum and defined by its real permittivity $\varepsilon>\varepsilon_{0}$.

One wants now to show relation 3.53 . Using $\tilde{\mathrm{H}} 3$, one deduces by uniqueness of the solution of $(3.42)$ and 3.43$)$ in $W_{1,-1}\left(\mathbb{R}^{3}\right)$, that $V_{s}(\cdot,-\bar{\omega})$, the solution of these equations corresponding to an incident field $\overline{\mathbf{E}_{0}}$, is equal to $\overline{V_{s}(\cdot, \omega)}$ (where $V_{s}(\cdot, \omega)$ stands for the solution with incident field $\left.\mathbf{E}_{0}\right)$. Thus, it follows using $\tilde{\mathrm{H}} 3$ and the relations 3.41 and 3.51 that $\boldsymbol{\alpha}(-\bar{\omega})=\overline{\boldsymbol{\alpha}(\omega)}$. Finally, one deduces from this last equality that

$$
f(-\bar{\omega})=\overline{\boldsymbol{\alpha}(\omega)} \mathbf{E}_{0} \cdot \overline{\mathbf{E}_{0}} .
$$

In the particular case of a real-valued field $\mathbf{E}_{0}$, this implies directly the relation (3.53). Obtaining the same relation for non real-valued incident fields requires the symmetry of the polarizability tensor: $\boldsymbol{\alpha}(\omega)^{\top}=\boldsymbol{\alpha}(\omega)$ which is a consequence of the reciprocity principle $\tilde{\mathrm{H}} 5$ (for its proof see for instance [86] p. 62). This concludes the proof.

Finally, we have to show that $f$ satisfies the hypothesis H4. This is the purpose of the following proposition.

Proposition 25. For any fixed incident field $\mathbf{E}_{0} \in \mathbb{C}^{3}$, the function $f$ defined by (3.40) satisfies the hypothesis $\mathrm{H} 4$ :

$$
\operatorname{Im} f(\omega) \geq 0, \forall \omega \in \mathbb{R}^{+} .
$$

Moreover, if $\left[\omega_{-}, \omega_{+}\right]$is a transparency window, that is a frequency band for which $\operatorname{Im} \boldsymbol{\varepsilon}(\mathbf{x}, \cdot)=0$ for a. e. $\mathbf{x} \in \Omega \backslash \mathcal{O}$ then

$$
\operatorname{Im} f(\omega)=0, \forall \omega \in\left[\omega_{-}, \omega_{+}\right] .
$$

Proof. Let $\mathbf{E}_{0}$ be a fixed vector of $\mathbb{C}^{3}, \omega$ a non-negative frequency and $R$ a positive real number satisfying $R>R_{0}$. We denote by $\mathbf{E}$ the electrical field associated with the incident field $\mathbf{E}_{0}$ by the equation (3.37). Then, by using $\tilde{\mathrm{H}} 4$, one has that:

$$
I(R, \omega)=\int_{B(0, R)} \operatorname{Im} \boldsymbol{\epsilon}(\mathbf{x}, \omega) \mathbf{E}(\mathbf{x}, \omega) \cdot \overline{\mathbf{E}(\mathbf{x}, \omega)} \mathrm{d} \mathbf{x}=\operatorname{Im}\left(\int_{B(0, R)} \boldsymbol{\epsilon}(\mathbf{x}, \omega) \mathbf{E}(\mathbf{x}, \omega) \cdot \overline{\mathbf{E}(\mathbf{x}, \omega)} \mathrm{d} \mathbf{x}\right) \geq 0 .
$$


We point out that $I(R, \omega)$ is constant with respect to $R$ for $R>R_{0}$ since $\operatorname{Im} \epsilon(\cdot, \omega)$ is compactly supported in $\Omega \subset B(0, R)$. Using the Green identity and the facts that $\mathbf{E}=-\nabla \mathbf{V}$ is divergence free (see 3.37) and that $\boldsymbol{\varepsilon}(\mathbf{x}, \omega)=\varepsilon_{0} \mathbf{I}$ on $\partial B(0, R)$, one gets:

$$
I(R, \omega)=\operatorname{Im}\left(\int_{\partial B(0, R)} \mathbf{V}(\mathbf{x}, \omega) \cdot \varepsilon_{0} \overline{\mathbf{E}(\mathbf{x}, \omega) \cdot \mathbf{n}} \mathrm{d} \mathbf{x}\right) \geq 0 .
$$

From the asymptotics 3.38 of $\mathbf{V}(\mathbf{x}, \omega)$ and the asymptotics of the electric field

$$
\mathbf{E}(\mathbf{x}, \omega)=-\nabla V(\mathbf{x}, \omega)=\mathbf{E}_{0}-\frac{\mathbf{p}(\omega)}{4 \pi \varepsilon_{0}|\mathbf{x}|^{3}}+3 \frac{(\mathbf{p}(\omega) \cdot \mathbf{x}) \mathbf{x}}{4 \pi \varepsilon_{0}|\mathbf{x}|^{5}}+O\left(\frac{1}{|\mathbf{x}|^{4}}\right)
$$

one gets:

$$
I(R, \omega)=3 \operatorname{Im}\left(\int_{\partial B(0, R)} \frac{(\mathbf{p}(\omega) \cdot \mathbf{x})}{4 \pi \varepsilon_{0}|\mathbf{x}|^{4}} \cdot \overline{\mathbf{E}_{0} \cdot \mathbf{x}} \mathrm{d} \mathbf{x}\right)+O\left(\frac{1}{|\mathbf{x}|}\right) \geq 0 .
$$

Using the fact that $\mathbf{p}(\omega)=\boldsymbol{\alpha}(\omega) \mathbf{E}_{0}$ leads to

$$
\operatorname{Im}\left(\int_{\partial B(0, R)} \frac{\left(\boldsymbol{\alpha}(\omega) \mathbf{E}_{0} \cdot \mathbf{x}\right)}{4 \pi \varepsilon_{0}|\mathbf{x}|^{4}} \cdot \overline{\mathbf{E}_{0} \cdot \mathbf{x}} \mathrm{d} \mathbf{x}\right) \geq 0 .
$$

By virtue of the algebraic identity

$$
\left(\boldsymbol{\alpha}(\omega) \mathbf{E}_{0} \cdot \mathbf{x}\right) \cdot \overline{\mathbf{E}_{0} \cdot \mathbf{x}}=\mathbf{x} \mathbf{x}^{\top} \boldsymbol{\alpha}(\omega) \mathbf{E}_{0} \cdot \overline{\mathbf{E}_{0}}
$$

one finally obtains

$$
\operatorname{Im}\left(\int_{\partial B(0, R)} \frac{\mathbf{x} \mathbf{x}^{\top} \boldsymbol{\alpha}(\omega) \mathbf{E}_{0} \cdot \overline{\mathbf{E}_{0}}}{4 \pi \varepsilon_{0}|\mathbf{x}|^{4}} \mathrm{~d} \mathbf{x}\right)=\frac{1}{3 \varepsilon_{0}} \operatorname{Im}\left(\boldsymbol{\alpha}(\omega) \mathbf{E}_{0} \cdot \overline{\mathbf{E}_{0}}\right)=\frac{1}{3 \varepsilon_{0}} \operatorname{Im} f(\omega) \geq 0 .
$$

(to derive the last inequality, we use the identity: $\int_{\partial B(0, R)} \mathbf{x x}^{\top} \mathrm{d} \mathbf{x}=4 \pi / 3 \mathbf{I}$ which can be shown by a straightforward computation).

In the particular case where $\operatorname{Im} \varepsilon(\mathbf{x}, \omega)=0$ for a. e. $\mathbf{x} \in \Omega \backslash \mathcal{O}$, one has $I(R, \omega)=0$ and thus one deduces that $\operatorname{Im} f(\omega)=0$. This concludes the proof.

One concludes this subsection by the following theorem which defines a Herglotz function associated with the polarizability tensor.

Theorem 26. Let $\mathbf{E}_{0}$ be a non-zero fixed vector of $\mathbb{C}^{3}$ and $f$ the function defined by (3.40). If the cloak $\Omega \backslash \mathcal{O}$ satisfies the hypotheses $\tilde{\mathrm{H}} 1-7$, then

$$
v(\omega)=\omega f(\sqrt{\omega})=\omega \boldsymbol{\alpha}(\sqrt{\omega}) \mathbf{E}_{0} \cdot \overline{\mathbf{E}_{0}}
$$

is a Herglotz function which is analytic on $\mathbb{C} \backslash \mathbb{R}^{+}$and negative on $\mathbb{R}^{-*}$. Moreover, in its representation given by the Theorem 2, the measure $\mathrm{m}$ is supported in $\mathbb{R}^{+}$and $\alpha$ is equal to $f_{\infty}=\boldsymbol{\alpha}(\infty) \mathbf{E}_{0} \cdot \overline{\mathbf{E}_{0}}$.

Proof. The proof is an immediate consequence of propositions 22, 24 and 25 which show that the function $f$ satisfies the hypotheses H1-4 and thus the function $v$ defined by (3.54) satisfies the Corollary 10.

Remark 27. As the reciprocity principle $\tilde{\mathrm{H}} 5$ is only required to prove $\mathrm{H} 3$ for non real incident fields $\mathbf{E}_{0}$ (see Proposition 24), Theorem 26 still holds for non-reciprocal media under the additional condition that $\mathbf{E}_{0} \in \mathbb{R}^{3}$. 


\subsection{Fundamental limits of broadband passive cloaking in quasi-statics}

\subsubsection{General bounds on the polarizability tensors}

One assumes in the following that our cloak satisfies the hypotheses $\tilde{\mathrm{H}} 1-7$. One wants first to establish that the analyticity property of the polarizability tensor is sufficient to prove that $\boldsymbol{\alpha}$ does not vanish on the whole frequency band $\left[\omega_{-}, \omega_{+}\right]$. Indeed, for any fixed non zero incident field $\mathbf{E}_{0} \in \mathbb{C}^{3}$, the function $f$ defined by 3.40 is analytic on $\mathbb{C}^{+}$and continuous on $\mathrm{cl} \mathbb{C}^{+}$. Thus, if by contradiction $\boldsymbol{\alpha}$ vanishes on $\left[\omega_{-}, \omega_{+}\right]$, so does $f$. Then, using the Schwarz reflection principle and the analytic continuation, one deduces that $f$ vanishes also on the whole upperhalf plane $\mathbb{C}^{+}$, which contradicts the fact that $f$ tends to $f_{\infty}>0$, when $|\omega| \rightarrow \infty$. However, such analytic continuation arguments are not of practical interest. Indeed, it is possible for instance for a polynomial to be arbitrary close to 0 on one disk and arbitrarily closed to 1 on another disjoint disk (see [30]). But, knowing that one can construct a Herglotz function associated with $f$ (see Theorem 26) gives us meaningful inequalities by using the bounds derived in section 2 . These inequalities establish fundamental limits on the cloaking effect over a frequency band $\left[\omega_{-}, \omega_{+}\right]$, that we present in this subsection.

For any non-zero incident field $\mathbf{E}_{0} \in \mathbb{C}^{3}$, the function $v$ defined by (3.54) satisfies the bound 2.23). In the particular case of Dirac measures: $m=\delta_{\xi}, \xi \in \mathbb{R}$, which optimizes the right hand side of (2.23) (see Theorem 14), this inequality becomes the bound (2.26), that we are recalling here in the case of our cloaking application:

$$
\lim _{y \rightarrow 0^{+}} \int_{x_{-}}^{x_{+}} \operatorname{Im}\left(\frac{1}{\xi-(x+\mathrm{i} y) \boldsymbol{\alpha}(\sqrt{x+\mathrm{i} y}) \mathbf{E}_{0} \cdot \overline{\mathbf{E}_{0}}}\right) \mathrm{d} x \leq \frac{\pi}{\boldsymbol{\alpha}(\infty) \mathbf{E}_{0} \cdot \overline{\mathbf{E}_{0}}}, \forall \xi \in \mathbb{R}
$$

which holds for any interval $\left[x_{-}, x_{+}\right]$of $\mathbb{R}^{+*}$. One notices that the geometry and the dielectric contrast of the inclusion $\mathcal{O}$ are encoded in the expression of the polarizability tensor $\boldsymbol{\alpha}(\infty)$. For instance, in the case of circular inclusion of radius $R$, one has

$$
\boldsymbol{\alpha}(\infty)=4 \pi R^{3} \varepsilon_{0} \frac{\varepsilon-\varepsilon_{0}}{\varepsilon+2 \varepsilon_{0}} \mathbf{I} .
$$

Explicit expressions of $\boldsymbol{\alpha}(\infty)$ can be also derived for ellipsoidal shapes (see for instance [81, 87]).

\subsubsection{The case of a transparency window}

We are now interested in deriving a more explicit version of the bound 3.55$)$ in the case where $\left[\omega_{-}, \omega_{+}\right]$is a transparency window, that is a frequency band for which $\operatorname{Im} \varepsilon(\mathbf{x}, \cdot)=0$ for a. e. $\mathbf{x}$ in $\Omega \backslash \mathcal{O}$. In other words, we assume that the cloak material is composed of a material that one can consider lossless in this frequency range. In particular, this latter condition implies that the polarizability tensor satisfies also $\operatorname{Im} \boldsymbol{\alpha}(\omega)=0$ on $\left[\omega_{-}, \omega_{+}\right]$(see Proposition 25). Thus, one can directly apply the bound (2.28) derived in Proposition 15 to obtain

$$
\omega_{0}^{2}\left(\boldsymbol{\alpha}\left(\omega_{0}\right)-\boldsymbol{\alpha}(\infty)\right) \mathbf{E}_{0} \cdot \overline{\mathbf{E}_{0}} \leq \omega^{2}(\boldsymbol{\alpha}(\omega)-\boldsymbol{\alpha}(\infty)) \mathbf{E}_{0} \cdot \overline{\mathbf{E}_{0}}, \forall \omega, \omega_{0} \in\left[\omega_{-}, \omega_{+}\right] \text {such that } \omega_{0} \leq \omega
$$

As, this last inequality holds for any $\mathbf{E}_{0} \in \mathbb{C}^{3}$, one deduces that

$$
\omega_{0}^{2}\left(\boldsymbol{\alpha}\left(\omega_{0}\right)-\boldsymbol{\alpha}(\infty)\right) \leq \omega^{2}(\boldsymbol{\alpha}(\omega)-\boldsymbol{\alpha}(\infty)), \forall \omega, \omega_{0} \in\left[\omega_{-}, \omega_{+}\right] \text {such that } \omega_{0} \leq \omega
$$


which is to be interpreted as a matrix inequality ( $\mathbf{A} \leq \mathbf{B}$ if and only if $\mathbf{B}-\mathbf{A}$ is positive semidefinite). We want to emphasize that this bound is sharp in the sense that there exists an analytic function $\boldsymbol{\alpha}$ (given by a Drude type model) such that $f(\cdot)=\boldsymbol{\alpha}(\cdot) \mathbf{E}_{0} \cdot \overline{\mathbf{E}_{0}}$ satisfies the properties H1-4 (except the continuity at $\omega=0$ ) for any $\mathbf{E}_{0} \in \mathbb{C}^{3}$, namely

$$
\boldsymbol{\alpha}(\omega)=\boldsymbol{\alpha}(\infty)-\frac{\omega_{0}^{2}\left[\boldsymbol{\alpha}(\infty)-\boldsymbol{\alpha}\left(\omega_{0}\right)\right]}{\omega^{2}} \text { with } \boldsymbol{\alpha}\left(\omega_{0}\right) \leq \boldsymbol{\alpha}(\infty)
$$

for which one has equality in (3.57). This function is singular at $\omega=0$, so it does not satisfy completely the hypothesis H1, but the continuity assumption on the real line in $\mathrm{H} 1$ can be weakened as we point out in Remarks 11 and 18 .

Now coming back to our initial cloaking problem, if one can cloak the inclusion at one frequency $\omega_{0} \in\left[\omega_{-}, \omega_{+}\right]$, and thus if $\boldsymbol{\alpha}\left(\omega_{0}\right)=0$ then the bound (3.57) implies

$$
\begin{aligned}
\boldsymbol{\alpha}(\omega) & \leq-\boldsymbol{\alpha}(\infty) \frac{\omega_{0}^{2}-\omega^{2}}{\omega^{2}} \text { if } \omega_{-} \leq \omega \leq \omega_{0} \\
& \geq \boldsymbol{\alpha}(\infty) \frac{\omega^{2}-\omega_{0}^{2}}{\omega^{2}} \text { if } \omega_{0} \leq \omega \leq \omega_{+},
\end{aligned}
$$

which obviously forces $\boldsymbol{\alpha}(\omega)$ to be non-zero away from the frequency $\omega_{0}$ (provided one is still in the transparency window $\left[\omega_{-}, \omega_{+}\right]$where there is no absorption). Thus, one cannot achieve broad band passive cloaking in a transparency window.

\subsubsection{The lossy case}

The bound (3.57) is only valid if $\left[\omega^{-}, \omega^{+}\right]$is a transparency window and thus does not hold if the cloak is a lossy material over this frequency range. Nevertheless, for a lossy cloak, one can apply the bounds (2.34), 2.35), (2.36) derived in section 2.6 to the function $f$. In particular, the bound (2.36) takes the form:

$$
\frac{1}{4}\left(\omega_{+}^{2}-\omega_{-}^{2}\right) \boldsymbol{\alpha}(\infty) \mathbf{E}_{0} \cdot \overline{\mathbf{E}_{0}} \leq \max _{x \in\left[\omega_{-}, \omega_{+}\right]}\left|\omega^{2} \boldsymbol{\alpha}(\omega) \mathbf{E}_{0} \cdot \overline{\mathbf{E}_{0}}\right|, \forall \mathbf{E}_{0} \in \mathbb{C}^{3}
$$

This bound gives a limitation to the cloaking effect by controlling from below the maximum of the function $\omega \rightarrow \omega^{2} \boldsymbol{\alpha}(\omega) \mathbf{E}_{0} \cdot \overline{\mathbf{E}_{0}}$ by a positive quantity depending both on the frequency bandwidth: $\omega_{+}-\omega_{-}$and on the geometry and the dielectric contrast of the inclusion with the term $\boldsymbol{\alpha}(\infty) \mathbf{E}_{0} \cdot \overline{\mathbf{E}_{0}}$. However, from an experimental perspective the more general bounds (2.34) or 2.35) are more meaningful since the value of the left hand side of 3.58 would be drastically changed if there was an extremely narrow resonant spike in $f$ in the considering interval and such a spike would be difficult to experimentally detect.

Remark 28. For the sake of generality, we point out that all the bounds derived in this subsection, with the exception of (3.57), which does not hold at the tensor level (but only at the scalar level (3.56)) are still satisfied for real-valued incident fields $\mathbf{E}_{0}$ if the medium does not satisfy the reciprocity principle $\tilde{\mathrm{H}} 5$. Thus, one has proved also that one cannot achieve broadband cloaking with non reciprocal materials. 


\section{Acknowledgements}

G.W. Milton is grateful to the Mittag-Leffler Institute for hosting his visit to Sweden during the program on Inverse Problems and Applications, where this work was initiated, and both authors are grateful to the National Science Foundation for support through grant DMS-1211359. Additionally, they are grateful to the Institute for Mathematics and its applications in Minneapolis for supporting their visit there in the Fall 2016.

\section{References}

[1] A. Alú And N. Engheta, Achieving transparency with plasmonic and metamaterial coatings, Physical Review E (Statistical physics, plasmas, fluids, and related interdisciplinary topics), 72 (2005), p. 0166623.

[2] — Plasmonic and metamaterial cloaking: physical mechanisms and potentials, Journal of Optics A: Pure and Applied Optics, 10 (2008), p. 093002.

[3] H. Ammari, G. Ciraolo, H. Kang, H. Lee, and G. W. Milton, Anomalous localized resonance using a folded geometry in three dimensions, Proceedings of the Royal Society A: Mathematical, Physical, \& Engineering Sciences, 469 (2013), p. 20130048. Also available as arXiv:1301.5712 [math-ph].

[4] _ Spectral theory of a Neumann-Poincaré-type operator and analysis of cloaking due to anomalous localized resonance, Archive for Rational Mechanics and Analysis, 208 (2013), pp. 667-692. See also arXiv:1109.0479 [math.AP].

[5] — Spectral theory of a Neumann-Poincaré-type operator and analysis of cloaking due to anomalous localized resonance II, Contemporary Mathematics, 615 (2014), pp. 1-14.

[6] H. Ammari And H. Kang, Polarization and moment tensors: with applications to inverse problems and effective medium theory, vol. 162, Springer Science \& Business Media, New York, 2007.

[7] K. Ando, Y.-G. Ji, H. KAnG, K. Kim, And S. Yu, Spectral properties of the neumannpoincaré operator and cloaking by anomalous localized resonance for the elasto-static system, (2015). Submitted. Available as arXiv:1510.00989 [math.AP].

[8] G. A. Baker JR. And P. R. Graves-Morris, Padé Approximants: Basic Theory. Part I. Extensions and Applications. Part II, vol. 13 \& 14 of Encyclopedia of Mathematics and its Applications, Addison-Wesley, Reading, Massachusetts, 1981.

[9] C. Berg, Stieltjes-Pick-Bernstein-Schoenberg and their connection to complete monotonicity, in Positive Definite Functions: From Schoenberg to Space-Time Challenges, J. Mateu and E. Porcu, eds., Editorial Universitat Jaume I, Department of Mathematics, Castellón de la Plana, Spain, 2008, pp. 15-45.

[10] D. J. Bergman, Analytical properties of the complex effective dielectric constant of a composite medium with applications to the derivation of rigorous bounds and to percolation 
problems, in Electrical Transport and Optical Properties of Inhomogeneous Media, J. C. Garland and D. B. Tanner, eds., vol. 40 of AIP Conference Proceedings, Woodbury, New York, 1978, American Institute of Physics, pp. 46-61.

[11] A. Bernland, A. Luger, And M. Gustafsson, Sum rules and constraints on passive systems, Technical Report LUTEDX/(TEAT-7193)/1-31/(2010), 2010.

[12] A. Bernland, A. Luger, And M. Gustafsson, Sum rules and constraints on passive systems, Journal of Physics A: Mathematical and Theoretical, 44 (2011), p. 145205.

[13] C. Bonifasi-Lista, E. Cherkaev, and Y. Yeni, Analytical approach to recovering bone porosity from effective complex shear modulus., Journal of biomechanical engineering, 13 (2009), p. 121003.

[14] A. Bonnet-Bendhia, L. Chesnel, And P. C. JR., T-coercivity for scalar interface problems between dielectrics and metamaterials, Mathematical Modelling and Numerical Analysis, 46 (2012), pp. 1363-1387.

[15] G. Bouchitté And B. Schweizer, Cloaking of small objects by anomalous localized resonance, Quarterly Journal of Mechanics and Applied Mathematics, 63 (2010), pp. 437463.

[16] O. P. Bruno And S. Lintner, Superlens-cloaking of small dielectric bodies in the quasistatic regime, Journal of Applied Physics, 102 (2007), p. 124502.

[17] M. Cassier, C. Hazard, And P. Joly, Spectral theory for maxwell's equations at the interface of a metamaterial. Part I: Generalized Fourier transform., available online on Arxiv at https://128.84.21.199/abs/1610.03021, (2016).

[18] M. Cessenat, Mathematical Methods in Electromagnetism: Linear Theory and Applications, vol. 41 of Series on advances in mathematics for applied sciences, World Scientific Publishing Co., Singapore / Philadelphia / River Edge, New Jersey, 1996.

[19] Y. T. Christodoulides and D. B. Pearson, Generalized value distribution for Herglotz functions and spectral theory., Mathematical Physics, Analysis and Geometry, 7 (2004), pp. 309-331.

[20] R. Dautray And J. L. Lions, Mathematical Analysis and Numerical Methods for Science and Technology: Volume 1 Physical Origins and Classical Methods., Springer-Verlag, Berlin, 2000.

[21] L. S. Dolin, To the possibility of comparison of three-dimensional electromagnetic systems with nonuniform anisotropic filling, Izvestiya Vysshikh Uchebnykh Zavedeniı. Radiofizika (see http://www.math.utah.edu/ milton/DolinTrans2.pdf for an english translation of Dolin's paper), 4 (1961), pp. 964-967.

[22] L. Evans, Partial Differential Equations, American Mathematical Society, Providence, 2008. 
[23] F. Gesztesy and E. Tsekanovskit, On matrix-valued Herglotz functions, Mathematische Nachrichten, 218 (2000), pp. 61-138.

[24] K. Golden and G. Papanicolaou, Bounds for effective parameters of heterogeneous media by analytic continuation, Communications in Mathematical Physics, 90 (1983), pp. 473-491.

[25] A. Greenleaf, Y. Kurylev, M. Lassas, and G. Uhlmann, Cloaking devices, electromagnetic wormholes, and transformation optics, SIAM Review, 51 (2009), pp. 3-33.

[26] A. Greenleaf, M. Lassas, and G. Uhlmann, Anisotropic conductivities that cannot be detected by EIT, Physiological Measurement, 24 (2003), pp. 413-419.

[27] — On non-uniqueness for Calderón's inverse problem, Mathematical Research Letters, 10 (2003), pp. 685-693.

[28] F. Guevara Vasquez, G. W. Milton, and D. Onofrei, Active exterior cloaking for the 2D Laplace and Helmholtz equations, Physical Review Letters, 103 (2009), p. 073901.

[29] — Broadband exterior cloaking, Optics Express, 17 (2009), pp. 14800-14805.

[30] _ Mathematical analysis of the two dimensional active exterior cloaking in the quasistatic regime, Analysis and Mathematical Physics, 2 (2012), pp. 231-246.

[31] M. Gustafsson And D. SjöBerg, Sum rules and physical bounds on passive metamaterials, New Journal of Physics, 12 (2010), p. 043046.

[32] M. Gustafsson And D. SJÖBerg, Time-domain approach to the forward scattering sum rule, Proceedings of the Royal Society A, 466 (2010), pp. 579-3592.

[33] H. Hashemi, C.-W. Qiu, A. P. McCauley, J. D. Joannopoulos, and S. G. JohnSON, Diameter-bandwidth product limitation of isolated-object cloaking, Physical Review A, 86 (2012), p. 013804.

[34] P. Henrici, Applied and computational complex analysis, discrete Fourier analysis, Cauchy integrals, construction of conformal maps, univalent functions, vol. 3, John Wiley \& Sons, 1993.

[35] J. D. Jackson, Classical Electrodynamics, John Wiley and Sons, New York, NY, third ed., 1999.

[36] H. Kang And G. W. Milton, Solutions to the Pólya-Szegö conjecture and the Weak Eshelby Conjecture, Archive for Rational Mechanics and Analysis, 188 (2008), pp. 93-116.

[37] T. Kato, Perturbation Theory for Linear Operators, Classics in Mathematics, SpringerVerlag, Berlin, Germany / Heidelberg, Germany / London, UK / etc., 1995.

[38] M. Kerker, Invisible bodies, Journal of the Optical Society of America, 65 (1975), pp. 376379. 
[39] H. Kettunen, M. Lassas, And P. Ola, On absence and existence of the anomalous localized resonance without the quasi-static approximation, (2014). Submitted. Available as arXiv:1406.6224 [math-ph].

[40] R. V. Kohn, J. Lu, B. Schweizer, And M. I. Weinstein, A variational perspective on cloaking by anomalous localized resonance, Communications in Mathematical Physics, 328 (2014), pp. 1-27. Available as arXiv:1210.4823 [math.AP].

[41] R. V. Kohn and M. S. Vogelius, Inverse problems, in Proceedings of the Symposium in Applied Mathematics of the American Mathematical Society and the Society for Industrial and Applied Mathematics, New York, April 12-13, 1983, D. W. McLaughlin, ed., vol. 14 of SIAM AMS Proceedings, Providence, RI, USA, 1984, American Mathematical Society, pp. 113-123.

[42] Y. Lai, H. Chen, Z.-Q. Zhang, and C. T. Chan, Complementary media invisibility cloak that cloaks objects at a distance outside the cloaking shell, Physical Review Letters, 102 (2009), p. 093901.

[43] L. D. Landau, E. M. Lifshitz, and L. P. PitaevskiI , Electrodynamics of Continuous Media, vol. 8 of Landau and Lifshitz Course of Theoretical Physics, Elsevier ButterworthHeinemann, Oxford, UK, second ed., 1984.

[44] U. Leonhardt, Optical conformal mapping, Science, 312 (2006), pp. 1777-1780.

[45] U. Leonhardt and T. Tyc, Broadband invisibility by non-Euclidean cloaking, Science, 323 (2009), pp. 110-112.

[46] H. LI, J. LI, AND H. LiU, On quasi-static cloaking due to anomalous localized resonance in $\mathbb{R}^{3}$, SIAM Journal on Applied Mathematics, 75 (2016), pp. 1245-1260.

[47] Ø. Lind-Johansen, K. Seip, And J. SkaAR, The perfect lens on a finite bandwidth, Journal of Mathematical Physics, 50 (2009), p. 012908.

[48] L. Mattner, Complex differentiation under the integral, Nieuw Archief voor Wiskunde (Groningen), 5/2 (2001), pp. 32-35.

[49] R. C. McPhedran, N.-A. P. Nicorovici, L. C. Botten, and G. W. Milton, Cloaking by plasmonic resonance among systems of particles: cooperation or combat?, Comptes Rendus Physique, 10 (2009), pp. 391-399.

[50] T. Meklachi, G. W. Milton, D. Onofrei, A. E. Thaler, and G. Funchess, Sensitivity of anomalous localized resonance phenomena with respect to dissipation, Quarterly of Applied Mathematics, 74 (2016), pp. 201-234.

[51] D. A. B. Miller, On perfect cloaking, Optics Express, 14 (2006), pp. 12457-12466.

[52] O. D. Miller, C. W. Hsu, M. T. H. Reid, W. Qiu, B. G. Delacy, J. D. Joannopoulos, M. Soljačić, and S. G. Johnson, Fundamental limits to extinction by metallic nanoparticles, Physical Review Letters, 112 (2014), p. 123903. 
[53] G. W. Milton, Theoretical studies of the transport properties of inhomogeneous media, Unpublished report TP/79/1, University of Sydney, Sydney, Australia, 1979. Unpublished report. (Available on request from the author).

[54] _ Bounds on the complex permittivity of a two-component composite material, Journal of Applied Physics, 52 (1981), pp. 5286-5293.

[55] G. W. Milton, The Theory of Composites, vol. 6 of Cambridge Monographs on Applied and Computational Mathematics, Cambridge University Press, Cambridge, UK, 2002, pp. 295-298. Series editors: P. G. Ciarlet, A. Iserles, Robert V. Kohn, and M. H. Wright.

[56] G. W. Milton, D. J. Eyre, and J. V. Mantese, Finite frequency range KramersKronig relations: Bounds on the dispersion, Physical Review Letters, 79 (1997), pp. 30623065.

[57] G. W. Milton And N.-A. P. Nicorovici, On the cloaking effects associated with anomalous localized resonance, Proceedings of the Royal Society A: Mathematical, Physical, \& Engineering Sciences, 462 (2006), pp. 3027-3059.

[58] G. W. Milton, N.-A. P. Nicorovici, R. C. McPhedran, K. Cherednichenko, AND Z. JACOB, Solutions in folded geometries, and associated cloaking due to anomalous resonance, New Journal of Physics, 10 (2008), p. 115021.

[59] F. Monticone And A. Alú, Physical bounds on electromagnetic invisibility and the potential of superconducting cloaks, Photonics and Nanostructures - Fundamentals and Applications, Special issue for metamaterials, 12 (2014), pp. 330-339.

[60] _ Invisibility exposed: physical bounds on passive cloaking, Optica, 3 (2016), pp. $718-$ 724.

[61] J.-C. Nedelec, Acoustic and Electromagnetic Equations: Integral Representations for Harmonic Problems, vol. 144 of Applied Mathematical Sciences, Springer Science \& Business Media, New York, NY, 2001.

[62] R. Nevanlinna, Asymptotische Entwicklungen das Stieltjessche Momentenproblem, Annales Academiae Scientiarum Fennicae, Series A, 18 (1922).

[63] H.-M. NGUYÊN, Cloaking via anomalous localized resonance for doubly complementary media in the quasistatic regime, Journal Of The European Mathematical Society, 17 (2015), pp. 1327-1365.

[64] _ Cloaking an arbitrary object via anomalous localized resonance: the cloak is independent of the object., (2016). Available as arXiv:1607.06492.

[65] _ Cloaking via anomalous localized resonance for doubly complementary media in the finite frequency regime, (2016). Available as arXiv:1511.08053 [math.AP].

[66] H.-M. NGuYen, Limiting absorption principle and well-posedness for the Helmholtz equation with sign changing coefficients, Journal de Mathématiques Pures et Appliquées, 106 (2016), pp. 342-374. 
[67] H.-M. NGuyên And L. H. NGuYÊN, Cloaking using complementary media for the Helmholtz equation and a three spheres inequality for second order elliptic equations, Transactions of The American Mathematical Society, Series B, 2 (2015), pp. 93-112.

[68] L. H. NGuYÊN, Cloaking using complementary media in the quasistatic regime, Annales de l'Institut Henri Poincaré. Analyse non linéaire, (2016). In press. Available online.

[69] N. A. Nicorovici, R. C. McPhedran, And G. W. Milton, Optical and dielectric properties of partially resonant composites, Physical Review B (Solid State), 49 (1994), pp. 8479-8482.

[70] N.-A. P. Nicorovici, R. C. McPhedran, And L. C. Botten, Relative local density of states and cloaking in finite clusters of coated cylinders, Waves in Random and Complex Media. Propagation, Scattering and Imaging, 21 (2011), pp. 248-277.

[71] N.-A. P. Nicorovici, R. C. McPhedran, S. Enoch, and G. Tayeb, Finite wavelength cloaking by plasmonic resonance, New Journal of Physics, 10 (2008), p. 115020.

[72] N.-A. P. Nicorovici, G. W. Milton, R. C. McPhedran, and L. C. Botten, Quasistatic cloaking of two-dimensional polarizable discrete systems by anomalous resonance, Optics Express, 15 (2007), pp. 6314-6323.

[73] A. N. Norris, Acoustic integrated extinction, Proceedings of the Royal Society of London. Series A, 471 (2015), p. 20150008.

[74] A. N. Norris, F. A. Amirkulova, and W. J. Parnel, Source amplitudes for active exterior cloaking, Inverse Problems, 28 (2012), p. 105002.

[75] A. N. Norris, F. A. Amirkulova, And W. J. Parnell, Active elastodynamic cloaking, Mathematics and Mechanics of Solids : MMS, 19 (2014), pp. 603-625.

[76] H. M. Nussenzveig, Causality and dispersion relations, Academic Press, New York, 1972.

[77] J. O’Neill, Ö. Selsil, R. C. McPhedran, A. B. Movchan, and N. V. Movchan, Active cloaking of inclusions for flexural waves in thin elastic plates, Quarterly Journal of Mechanics and Applied Mathematics, 68 (2015), pp. 263-288.

[78] J. O’Neill, Ö. Selsil, R. C. McPhedran, A. B. Movchan, N. V. Movchan, and C. H. MogGach, Active cloaking of resonant coated inclusions for waves in membranes and kirchhoff plates, Quarterly Journal of Mechanics and Applied Mathematics, 69 (2016), pp. $115-159$.

[79] D. OnOfReI, On the active manipulation of fields and applications: I. The quasistatic case, Inverse Problems, 28 (2012), p. 105009.

[80] D. Onofrei And A. E. Thaler, Anomalous localized resonance phenomena in the nonmagnetic, finite-frequency regime, (2016). Submitted. Available as arXiv:1605.08954 [math$\mathrm{ph}]$.

[81] J. A. Osborn, Demagnetizing factors of the general ellipsoid, Physical Review, 67 (1945), pp. 351-357. 
[82] H. L. PÉCSELI, Fluctuations in physical systems., Cambridge University Press, 2000.

[83] J. B. Pendry, D. Schurig, And D. R. Smith, Controlling electromagnetic fields, Science, 312 (2006), pp. 1780-1782.

[84] E. M. Purcell, On the absorption and emission of light by interstellar grains., The Astrophysical Journal, 158 (1969), pp. 433-440.

[85] M. Selvanayagam and G. V. Eleftheriades, An active electromagnetic cloak using the equivalence principle, IEEE Antennas and Wireless Propagation Letters, 11 (2012), pp. $1226-1229$.

[86] C. Sohl, Dispersion Relations in Scattering and Antenna Problems, Ph.D. thesis, available online at http://lup.lub.lu.se/search/record/1221227, Lund University, 2008.

[87] E. C. Stoner, The demagnetizing factors for ellipsoids, Philosophical Magazine, 36 (1945), pp. 803-820.

[88] A. TIP, Linear dispersive dielectrics as limits of Drude-Lorentz systems, Physical Review E (Statistical physics, plasmas, fluids, and related interdisciplinary topics), 69 (2004), p. 016610.

[89] V. G. Veselago, The electrodynamics of substances with simultaneously negative values of $\epsilon$ and $\mu$, Uspekhi Fizicheskikh Nauk, 92 (1967), pp. 517-526. English translation in Soviet Physics Uspekhi 10(4):509-514 (1968).

[90] A. T. Welters, Y. Avniel, And S. G. Johnson, Speed-of-light limitations in passive linear media, Physical Review A (Atomic, Molecular, and Optical Physics), 90 (2014), p. 023847.

[91] A. D. Yaghjian And T. B. Hansen, Plane-wave solutions to frequency-domain and timedomain scattering from magnetodielectric slabs, Physical Review E (Statistical physics, plasmas, fluids, and related interdisciplinary topics), 73 (2006), p. 046608.

[92] A. H. Zemanian, Realizability theory for continuous linear systems., Courier Corporation, 1972. 